\title{
Thermal transport due to liquid jet impingement on superhydrophobic surfaces with isotropic slip
}

\author{
Matthew Searle*, Daniel Maynes, Julie Crockett \\ Department of Mechanical Engineering, Brigham Young University, 435 Crabtree \\ Building, Provo, Utah 84602, USA
}

\begin{abstract}
This paper presents an analytical investigation of thermal transport due to a steady, laminar, axisymmetric liquid jet impinging normally on a superhydrophobic (SHPo) surface maintained at constant surface temperature. At the liquid-surface boundary of the spreading thin film, an isotropic hydrodynamic slip and temperature jump are imposed to approximate the SHPo surface boundary condition. Applying an integral analysis within the thin film results in a system of differential equations which are solved numerically to obtain local hydrodynamic and thermal boundary layer thicknesses, thin film height, and local and radially averaged heat flux. The classical smooth hydrophobic scenario with no-slip and no-temperature jump showed excellent agreement with previous differential analysis of the same problem. The influence of varying temperature jump length on the local Nusselt number was obtained over a range of Reynolds and Prandtl numbers. Increasing temperature jump length results in a dramatic decrease in the local thermal transport near the impingement point. The greatest decrease occurs at small temperature jump lengths. Further, local and average Nusselt numbers are less influenced by the Reynolds and Prandtl numbers as temperature jump length increases. Overall, variations in the temperature jump length exert much more influence than variations in the hydrodynamic slip length.

Keywords:

superhydrophobic surface, jet impingement, convective heat transfer, slip length, temperature jump length
\end{abstract}

\footnotetext{
${ }^{*}$ Corresponding author. Tel.: +1 8017083972

Email address: matthew.c.searle@byu.edu (Matthew Searle)
}

Preprint submitted to International Journal of Heat and Mass Transfer March 13, 2017 


\section{Nomenclature}

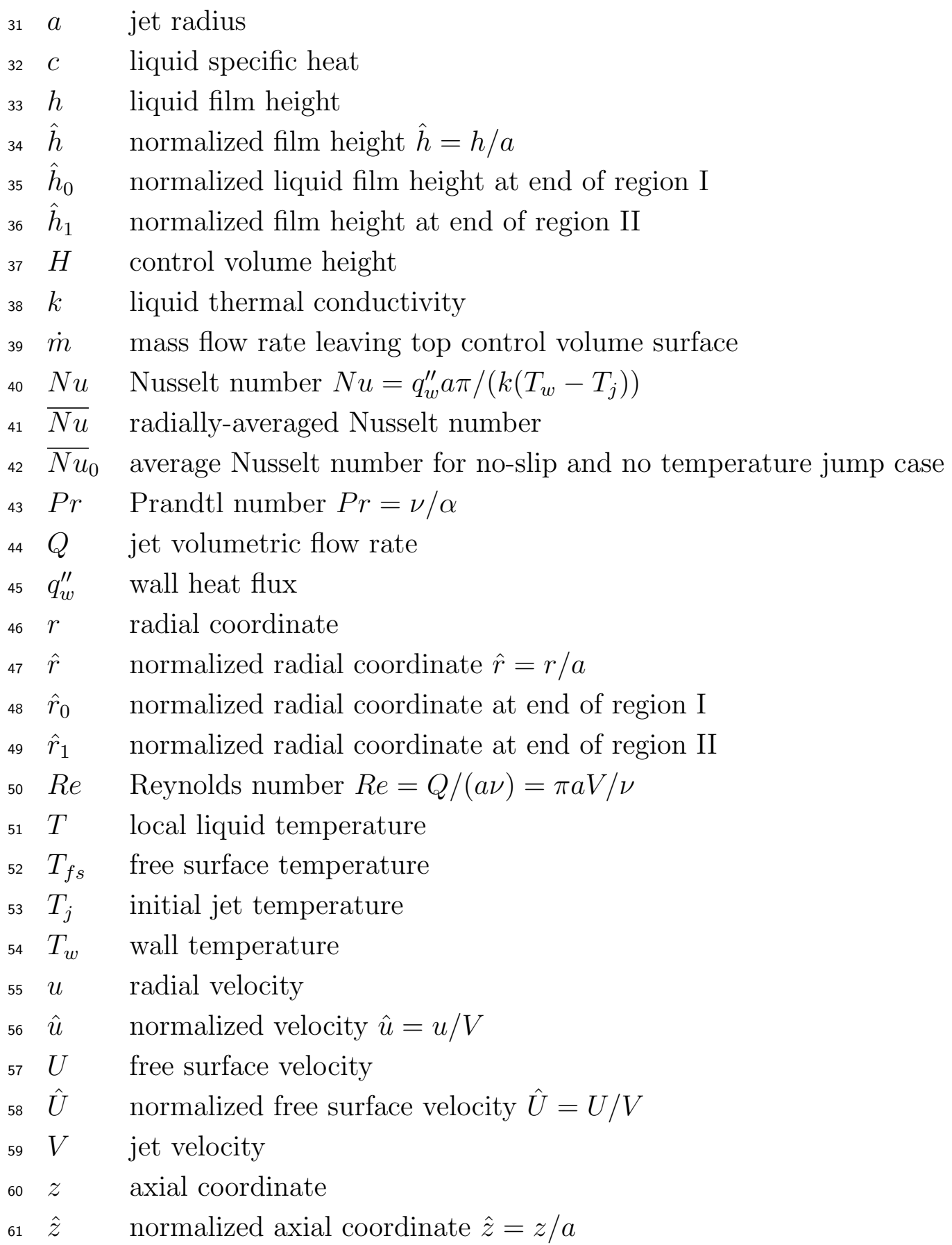


63

\section{Introduction}

Numerous studies have demonstrated drag reduction and self-cleaning behavior on superhydrophobic (SHPo) surfaces [1-7]. More recently, thermal transport physics have been explored as well. For a liquid flowing over a SHPo surface, thermal transport is inhibited due to insulating air-filled cavities at the wall. This has been observed in forced internal flow [8-11], natural convection in microchannels [12, 13], and Marangoni convection in droplets [14]. Boiling on SHPo surfaces is inhibited primarily due to an early transition 
from nucleate to film boiling resulting from the higher liquid-solid contact angle and altered surface geometry $[15,16]$. Conversely, condensation heat transfer is enhanced due to increased vapor-surface contact area [17, 18]. The focus of this paper is to explore the thermal transport for a liquid flow scenario which has not received attention: a laminar, liquid, axisymmetric jet impinging normally on a SHPo surface with isotropic slip.

The hydrodynamics of a jet impinging on a no-slip surface were modeled analytically by Watson [19]. These results specifically capture the development of the hydrodynamic boundary layer within the spreading thin film, the radial variation of thin film height, and estimate the hydrodynamic jump location. The model compares well with experimental data and has been improved to include surface tension in subsequent studies [20-22].

Thermal transport on a surface due to the spreading thin film of an impinging jet has also received significant prior attention. This interest has been motivated by industrial applications including cooling of electronic systems and quenching of metals and molded plastics [23, 24]. Analytical models for scenarios of constant wall temperature [25], constant wall heat flux [26], and varying wall temperature or heat flux [27, 28] have been accomplished. Additionally, numerical [29-33] and experimental studies [24, 34, 35] have been performed. In all studies, it was observed that the greatest thermal transport occurs near the impingement point and decreases asymptotically with increasing radial position. Additionally, the local and average Nusselt numbers increase with increasing Prandtl number and jet Reynolds number. SHPo surfaces differ significantly from smooth hydrophobic or hydrophilic surfaces. They are created by combining micro/nano-scale roughness with 
hydrophobic surface chemistry. Due to the surface hydrophobicity, water does not wet the cavities between roughness features if the Laplace pressure is not exceeded. Random or structured grid microfeatures are commonly employed. Important geometric parameters of SHPo surfaces with repeating features are the pitch, $w$, (the distance between microscale features) and cavity fraction, $F_{c}$ (ratio of the cavity area projected onto the interface to the total interface area).

If the air-filled cavities are on the micron scale, the hydrodynamic and thermal boundary conditions at a surface can be significantly altered $[17,36]$. Liquid near the wall encounters a no-slip boundary condition at the liquidsolid microfeature interface and a nearly shear-free boundary condition at the liquid-air interface between microfeatures. For macroscopic flows, it is beneficial to define an aggregate slip accounting for the alternating slip and no-slip boundary conditions to obtain a uniform boundary condition. This condition allows definition of a local slip velocity, $u_{s}$, which is proportional to the wall shear stress, $\tau_{w}$, and is given by, $u_{s}=\lambda \tau_{w} / \mu$, where $\mu$ is liquid dynamic viscosity, and $\lambda$ is the hydrodynamic slip length [36]. $\lambda$ is a property of the wall microstructure and can be defined in the Stokes flow regime by the surface parameters, cavity fraction and pitch [36].

The jet impingement problem on SHPo surfaces with isotropic [37] and anisotropic slip lengths [38] has previously been modeled analytically and validated experimentally [39]. Increasing $\lambda$ at fixed Reynolds number was observed to have a similar effect on the hydrodynamics as increasing the Reynolds number. The hydrodynamic boundary layer developed more slowly, the film thickness decreased, and the hydrodynamic jump radius increased. 
The composite liquid-solid and liquid-gas interface also alters the thermal boundary conditions. The liquid-solid interface yields a conventional convection boundary condition, while at the liquid-gas interface the heat flux is greatly reduced. Assuming metallic microfeatures (high thermal conductivity), the thermal conductivity of the gas filling each cavity is several orders of magnitude less than that of the microfeatures, rendering the liquid-gas interface nominally adiabatic. Again for macroscopic scale flows, an aggregate boundary condition can be defined in the form of a wall temperature jump, $\Delta T_{w}$. This temperature jump is proportional to the wall heat flux, $q_{w}^{\prime \prime}$, as given by the relationship, $\Delta T_{w}=\lambda_{T} q_{w}^{\prime \prime} / k$ [11], where $k$ is the liquid thermal conductivity and $\lambda_{T}$ the temperature jump length. The temperature jump length is the thermal analog to the hydrodynamic slip length $[9,11,40]$ and is a function of microscale feature geometry.

The relationship between $\lambda_{T}$ and $\lambda$ is an area of ongoing research. For Stokes flow between parallel plates with ribs aligned with the flow, $\lambda_{T}$ is very nearly equal to $\lambda$ [11]. A study of thermal transport in a channel with ribs oriented perpendicular to the flow observed a more complex relationship between $\lambda$ and $\lambda_{T}$ [8]. In this study, the ratio of $\lambda_{T}$ to $\lambda$ was shown to depend on Peclet number, relative module width (ratio of pitch to channel hydraulic diameter), and cavity fraction. At low Peclet number and small relative module width, the ratio of $\lambda_{T}$ to $\lambda$ is nominally 2. However, increasing the Peclet number to the order of 1,000 and setting the relative module width to 1 causes the ratio to decrease.

The effect of $\lambda_{T}$ on heat transfer in an impinging jet has not been previously explained, but is of interest as it represents a common cooling scenario 
for self-cleaning surfaces. Section 2 of this paper presents an integral analysis to quantify the effect of isotropic slip and temperature jump ( $\lambda$ and $\lambda_{T}$ ) on the local and average thermal transport. Results from the model are presented and discussed in Section 3 and conclusions are given in Section 4.

\section{Analysis}

\subsection{Model Description}

Shown in Fig. 1 is a schematic illustration of a vertical liquid jet impinging on a horizontal SHPo surface. A non-submerged, steady jet is assumed with jet velocity, $V$; radius, $a$; and flow rate, $Q$. Temperatures for the incoming jet and wall are $T_{j}$ and $T_{w}$, respectively, both of which are assumed to be uniform and $T_{w}>T_{j}$. The wall temperature jump is $\Delta T=T_{w}-T(r, z=0)$. A spatially uniform hydrodynamic slip length, $\lambda$, and temperature jump length, $\lambda_{T}$, characterize the superhydrophobic wall boundary condition.

For this analysis, $\hat{\lambda}$ and $\hat{\lambda}_{T}$ are assumed to be equal except in an exploratory case where they are varied independently. This assumption may be reasonable for typical SHPo surfaces but is an area for further research. As noted in the introduction, a recent study found $\hat{\lambda}$ and $\hat{\lambda}_{T}$ were approximately equal for a parallel plate channel with streamwise oriented ribs and cavities [11]. The text will discuss the influence of varying $\hat{\lambda}_{T}$ but, since $\hat{\lambda}=\hat{\lambda_{T}}$, the variation of $\hat{\lambda}$ is implied.

In Fig. 1, the development of the hydrodynamic $(\delta)$ and thermal $\left(\delta_{T}\right)$ boundary layers are illustrated. A coordinate system is fixed at the intersection of the jet centerline and the horizontal surface, where the z-axis is in the direction of the jet centerline, oriented normal to the surface and opposite 
the direction of flow. $r$ is the radial coordinate.

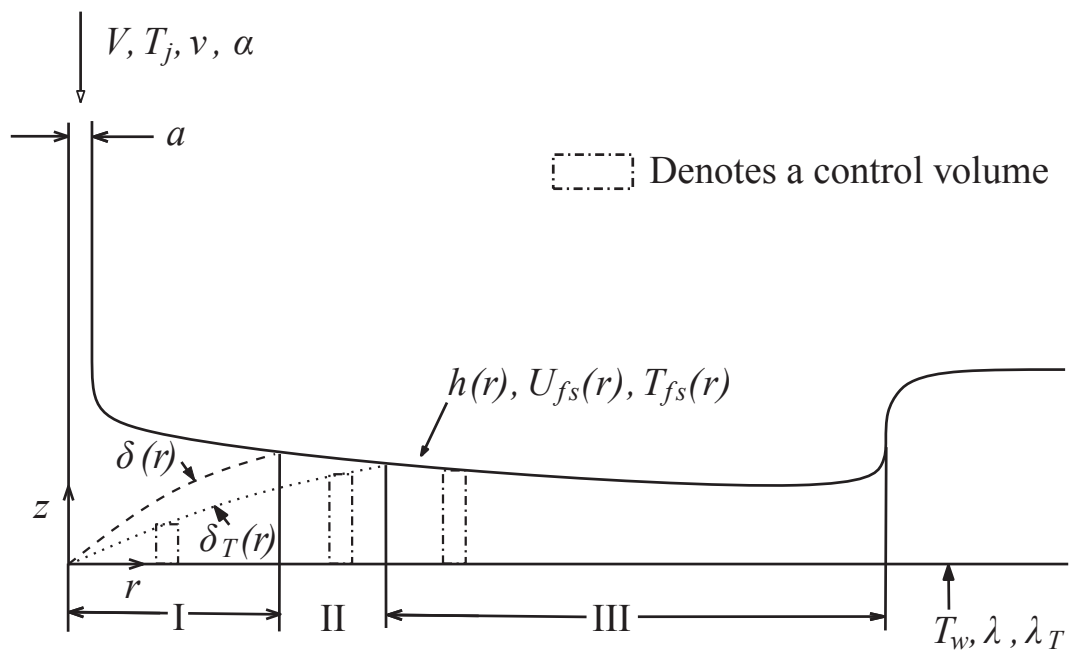

Figure 1: Schematic of a liquid jet impinging normally on a flat surface. The thin film is magnified along the z-coordinate to show the film dynamics. The film height (denoted by a solid line), the hydrodynamic boundary layer thickness (denoted by a dashed line), the thermal boundary layer thickness (denoted by a dotted line), and the control volumes utilized (denoted by dash-dotted lines) are shown.

Three regions exist moving radially outward from the impingement point prior to the location of a hydraulic jump. Region I spans $r$ between the impingement point and the location, $r_{0}$, where the hydrodynamic boundary layer, $\delta$, merges with the film height, $h$. Region II extends from $r_{0}$ up to the location where the thermal boundary layer, $\delta_{T}$, merges with the film height $\left(r_{1}\right)$. We note that $\delta_{T}<\delta$, since the jet is liquid and $\operatorname{Pr}>1$, where the Prandtl number takes the classical form, $\operatorname{Pr}=\nu / \alpha . \alpha$ is the liquid thermal diffusivity and $\nu$ is the liquid kinematic viscosity. Finally, region III spans $r$ between $r_{1}$ and the location where a hydraulic jump forms. 
Natural convection, radial conduction, viscous dissipation, and Marangoni convection are neglected in the analysis and constant liquid properties are assumed. Forced convection dominates natural convection due to the relatively high jet Reynolds numbers considered. Likewise, radial advection dominates radial conduction. Since the liquid has a moderate viscosity and relatively slow velocity, viscous dissipation is negligible. Due to the high jet Reynolds number, the influence of Marangoni convection is small since it is driven by variations in surface tension due to temperature variation across the meniscus between microfeatures. Heat transfer through the top liquid surface and shear stress between the water and air at this surface are also neglected and, following prior work, the initial thickness of the boundary layer was considered to be negligibly small [26].

\subsection{Application of Conservation Laws}

Conservation laws of mass and energy are applied to an annular control volume of thickness, $\Delta r$, and height, $H$. Control volume heights are selected such that known boundary conditions may be applied to the top control surface. Specifically, control volumes of height $\delta_{T}$ (regions I and II) and $h$ (region III) were used as shown in Fig. 1.

Applying conservation of mass and energy to a general control volume as shown in Fig. 1 yields

$$
\dot{m}=-2 \pi \rho \frac{\partial}{\partial r}\left(r \int_{0}^{H} u(r, z) \mathrm{d} z\right) \Delta r
$$


219

$$
-\dot{m} c T(r, H)+q_{w}^{\prime \prime}(2 \pi r) \Delta r-2 \pi \rho c \frac{\partial}{\partial r}\left(r \int_{0}^{H} u(r, z) T(r, z) \mathrm{d} z\right) \Delta r=0
$$

220

where $\dot{m}$ is the mass flow rate through the top surface of the control volume, $\rho$ is the liquid density, and $c$ is the liquid specific heat. As mentioned, the hydrodynamic problem was solved previously and is included here only as needed [37].

\subsection{Region $I$}

In region I, the hydrodynamic boundary layer develops until it merges with the film height. Throughout this region, the thermal boundary layer is developing as well, but with a thickness that is less than that of the hydrodynamic boundary layer. The control volume height is set to $H=\delta_{T}$. At this height, $T\left(r, \delta_{T}\right)=T_{j}$. Using these boundary conditions and substituting Eq. 1 into 2 yields

$$
q_{w}^{\prime \prime}=\frac{\rho c}{r} \frac{\partial}{\partial r}\left(r \int_{0}^{\delta_{T}} u\left(T(r, z)-T_{j}\right) \mathrm{d} z\right)
$$

A cubic velocity profile is assumed for region I, with a slip velocity boundary condition at $z=0$ and a free shear condition at $z=\delta$ and is expressed as

$$
u=\frac{V}{\frac{2}{3} \delta+\lambda}\left[\lambda+z-\frac{z^{3}}{3 \delta^{2}}\right]
$$

A cubic temperature profile is also assumed of the form

$$
T=a+b z+c z^{2}+d z^{3}
$$


236

237

238

244 Substituting Eq. 12 into Eq. 10 gives the following expression for the local 245 liquid temperature

Coefficients for this region I profile are found using the following boundary conditions

$$
T(r, z=0)=T_{w}-\Delta T_{w}
$$

$$
T\left(r, z=\delta_{T}\right)=T_{j}
$$

$$
\left.\frac{\partial T}{\partial z}\right|_{z=\delta_{T}}=0
$$

$$
\left.\frac{\partial^{2} T}{\partial z^{2}}\right|_{z=0}=0
$$

Applying these boundary conditions to Eq. 5 yields

$$
T=T_{w}-\Delta T_{w}-\frac{3\left(T_{w}-\Delta T_{w}-T_{j}\right)}{2 \delta_{T}} z+\frac{T_{s}-\Delta T_{w}-T_{j}}{2 \delta_{T}^{3}} z^{3}
$$

Using Fourier's law, the heat flux may be obtained from Eq. 5 as

$$
q_{w}^{\prime \prime}=-\left.k \frac{\mathrm{d} T}{\mathrm{~d} z}\right|_{z=0}=\frac{3 k\left(T_{w}-\Delta T_{w}-T_{j}\right)}{2 \delta_{T}}
$$

${ }_{21}$ The temperature jump length boundary condition [11] $q_{w}^{\prime \prime}=\left(\Delta T_{w} k\right) /\left(\lambda_{T}\right)$ is substituted into the assumed profile (Eq. 11) and solving for the temperature jump gives

$$
\Delta T_{w}=\frac{3 \lambda_{T}\left(T_{w}-T_{j}\right)}{2 \delta_{T}+3 \lambda_{T}}
$$

$$
T=T_{w}-\frac{3 \lambda_{T}\left(T_{w}-T_{j}\right)}{2 \delta_{T}+3 \lambda_{T}}-\frac{3\left(T_{w}-T_{j}\right)}{\left(2 \delta_{T}+3 \lambda_{T}\right)} z+\frac{T_{w}-T_{j}}{\delta_{T}^{2}\left(2 \delta_{T}+3 \lambda_{T}\right)} z^{3}
$$


The wall heat flux is determined by substituting the new temperature profile (Eq. 13) into Fourier's law and evaluating the derivative

$$
q_{w}^{\prime \prime}=\frac{3 k\left(T_{w}-T_{j}\right)}{2 \delta_{T}+3 \lambda_{T}}
$$

The velocity profile (Eq. 4), temperature profile (Eq. 13), and heat flux equation (Eq. 14) are all substituted into the energy equation (Eq. 3), and non-dimensionalized. Velocities are normalized by the jet velocity, $V$, and all lengths by the jet radius, $a$, with all non-dimensionalized variables denoted with a hat. The following nonlinear ordinary differential equation (ODE) describing the relationship between $\hat{\delta}_{T}, \hat{\delta}$, and $\hat{r}$ is obtained

$$
\frac{\pi \hat{r}}{\operatorname{RePr}}=\left(2 \hat{\delta}_{T}+3 \hat{\lambda}_{T}\right) \frac{d}{d \hat{r}}\left[\hat{r} \frac{\hat{\delta}_{T}^{2}\left(7 \hat{\delta}^{2}\left(4 \hat{\delta}_{T}+15 \hat{\lambda}\right)-2 \hat{\delta}_{T}^{3}\right)}{140 \hat{\delta}^{2}(2 \hat{\delta}+3 \hat{\lambda})\left(2 \hat{\delta}_{T}+3 \hat{\lambda}_{T}\right)}\right]
$$

where the Reynolds number is defined as $R e=Q /(a \nu)=\pi a V / \nu$.

In a similar manner, the following ODE was derived for the hydrodynamic variables in region I by substituting the assumed cubic velocity profile (Eq. 4) into the resulting momentum integral for a similar control volume to yield $[37]$

$$
\frac{\pi \hat{r}}{R e}=\left(\frac{2}{3} \hat{\delta}+\hat{\lambda}\right) \frac{d}{d \hat{r}}\left[\hat{r} \hat{\delta}^{2}\left(\frac{13}{210} \hat{\delta}+\frac{1}{4} \hat{\lambda}\right)\left(\frac{2}{3} \hat{\delta}+\hat{\lambda}\right)^{-2}\right]
$$

Applying conservation of mass (Eq. 1), the local film height, $\hat{h}$, as a function of $\hat{\lambda}$ and $\hat{\delta}$, can be expressed

$$
\hat{h}=\frac{1}{2 \hat{r}}+\frac{\hat{\delta}^{2}}{4\left(\frac{2}{3} \hat{\delta}+\hat{\lambda}\right)}
$$


A numerical solution for $\hat{h}, \hat{\delta}$ and $\hat{\delta}_{T}$ as a function of $\hat{r}$ was obtained by solving the system of equations formed by Eqs. 15, 16, and 17 with boundary conditions $\hat{\delta}(\hat{r}=0)=0$, and $\hat{\delta}_{T}(\hat{r}=0)=0$. The extent of region I was determined by numerically solving for the value of $\hat{r}$ where $\hat{\delta}$ reaches $\hat{h}$. Normalized values of the thermal boundary layer thickness and local film height at that point are labeled $\hat{\delta}_{T 0}$ and $\hat{h}_{0}$.

Nusselt number, $N u=q_{w}^{\prime \prime} a \pi /\left(k\left(T_{w}-T_{j}\right)\right)$, and the non-dimensional temperature jump, $\Delta \widehat{T}=\Delta T /\left(T_{w}-T_{j}\right)$, as functions of $\hat{r}$ may be obtained from the wall heat flux (Eq. 14) and the wall temperature jump (Eq. 12), respectively. These are given as Equations 18 and 19.

$$
\begin{aligned}
& N u=\frac{3 \pi}{2 \hat{\delta}_{T}+3 \hat{\lambda}_{T}} \\
& \Delta \widehat{T}=\frac{3 \hat{\lambda}_{T}}{2 \hat{\delta}_{T}+3 \hat{\lambda}_{T}}
\end{aligned}
$$

\section{Region II}

In region II, the hydrodynamic boundary layer has merged with the film height and the thermal boundary layer continues to grow until it also merges. The energy equation (Eq. 3), assumed temperature profile (Eq. 13), and the heat flux equation (Eq. 14) are the same as region I. However, boundary conditions on the velocity profile are altered in regions II and III as the free surface velocity is now variable. The resultant cubic velocity profile with a free shear boundary condition at the film thickness yields

$$
\hat{u}=\frac{\widehat{U}_{f s}}{\frac{2}{3} \hat{h}+\hat{\lambda}}\left[\hat{\lambda}+\hat{z}-\frac{\hat{z}^{3}}{3 \hat{h}^{2}}\right]
$$


where $\widehat{U}_{f s}$ is the normalized velocity at the free surface. $\widehat{U}_{f s}=U_{f s} / V$ may be expressed as

$$
\widehat{U}_{f s}=\frac{\frac{2}{3} \hat{h}+\hat{\lambda}}{2 \hat{h} \hat{r}\left(\frac{5}{12} \hat{h}+\hat{\lambda}\right)}
$$

which results from conservation of mass (Eq. 1). Substituting Eq. 21 into the velocity profile (Eq. 20) and then substituting this result and the temperature profile (Eq. 13) into the energy equation (Eq. 3) gives the following

$$
\frac{\pi \hat{r}}{\operatorname{RePr}}=\left(2 \hat{\delta}_{T}+3 \hat{\lambda}_{T}\right) \frac{d}{d \hat{r}}\left[\frac{\hat{\delta}_{T}^{2}\left(7 \hat{h}^{2}\left(4 \hat{\delta}_{T}+15 \hat{\lambda}\right)-2 \hat{\delta}_{T}^{3}\right)}{70 \hat{h}^{3}(5 \hat{h}+12 \hat{\lambda})\left(2 \hat{\delta}_{T}+3 \hat{\lambda}_{T}\right)}\right]
$$

The film thickness height can be determined throughout regions II and III by applying the integral momentum equation that is detailed in [37] and expanded as

$$
-\frac{2 \pi}{R e}=\hat{h}\left(\frac{5}{12} \hat{h}+\hat{\lambda}\right) \frac{d}{d \hat{r}}\left[\hat{r}^{-1} \hat{h}^{-1}\left(\frac{68}{315} \hat{h}^{2}+\frac{5}{6} \hat{h} \hat{\lambda}+\hat{\lambda}^{2}\right)\left(\frac{5}{12} \hat{h}+\hat{\lambda}\right)^{-2}\right]
$$

The system of equations formed by the hydrodynamic ODE (Eq. 23) and the energy ODE (Eq. 22) was solved numerically to obtain $\hat{h}$ and $\hat{\delta}_{T}$ as functions of $\hat{r}$ throughout region II. The initial conditions were $\hat{h}\left(\hat{r}=\hat{r}_{0}\right)=\hat{h}_{0}$ and $\hat{\delta}_{T}\left(\hat{r}=\hat{r}_{0}\right)=\hat{\delta}_{T 0}$. The end of region II was determined by numerically solving for the radial coordinate where $\hat{\delta}_{T}$ equaled $\hat{h}$. This radial coordinate was labeled $\hat{r}_{1}$ and the corresponding film height was labeled $\hat{h}_{1}$. The Nusselt number and $\Delta \widehat{T}$ as functions of $\hat{r}$ are defined in the same manner as in region 1 (Eq. 18 and Eq. 19). 
302

\section{Region III}

In region III, the hydrodynamic and thermal boundary layers have both merged with the film height. Here the control volume height is $H=h$ such that $\dot{m}=0$. Applying this simplification to the energy equation (Eq. 2) and rearranging yields

$$
q_{w}^{\prime \prime}=\frac{\rho c}{r} \frac{\partial}{\partial r}\left(r \int_{0}^{h} u T \mathrm{~d} z\right)
$$

In this region, the assumed velocity profile is the same as in region II (Eq. 20 ), but the boundary conditions for the temperature profile are now

$$
T(r, z=h)=T_{f s}(r)
$$

$$
\left.\frac{\partial T}{\partial z}\right|_{z=h}=0
$$

The assumed temperature profile is the same as region I and region II (Eq. 13) with the exception that $T_{j}$ is replaced by $T_{f s}(r)$ and $\delta_{T}$ by $h$

$$
T=T_{w}-\frac{3 \lambda_{T}\left(T_{w}-T_{f s}\right)}{2 h+3 \lambda_{T}}-\frac{3\left(T_{w}-T_{f s}\right)}{\left(2 h+3 \lambda_{T}\right)} z+\frac{T_{w}-T_{f s}}{h^{2}\left(2 h+3 \lambda_{T}\right)} z^{3}
$$

where $T_{f s}$ is the temperature at the free surface. Further, the heat flux equation (Eq. 14) and temperature jump equation (Eq. 12) are altered with the same substitutions

$$
q_{w}^{\prime \prime}=\frac{3 k\left(T_{w}-T_{f s}\right)}{2 h+3 \lambda_{T}}
$$

$$
\Delta T_{w}=\frac{3 \lambda_{T}\left(T_{w}-T_{f s}\right)}{2 h+3 \lambda_{T}}
$$


Using the assumed velocity (Eq. 20) and temperature (Eq. 27) profiles, as well as the expression for the free surface velocity (Eq. 21) in the governing equation (Eq. 24) results in the following form of the energy equation

$$
\frac{\pi \hat{r}}{\operatorname{RePr}}=\frac{2 \hat{h}+3 \hat{\lambda}}{\theta_{f s}} \frac{d}{d \hat{r}}\left[\frac{\hat{h} \theta_{f s}(26 \hat{h}+105 \hat{\lambda})}{70(5 \hat{h}+12 \hat{\lambda})\left(2 \hat{h}+3 \hat{\lambda}_{T}\right)}-\frac{\theta_{f s}}{6}\right]
$$

The non-dimensional temperature is defined as $\theta=\left(T-T_{w}\right) /\left(T_{j}-T_{w}\right)$ and the non-dimensional free surface temperature is $\theta_{f s}=\left(T_{f s}-T_{w}\right) /\left(T_{j}-T_{w}\right)$. As defined, the non-dimensional free surface temperature is unity when the free surface temperature is equal to the jet temperature and decreases to zero as the free surface temperature approaches the wall temperature.

The system of equations formed by the hydrodynamic ODE (Eq. 23) and energy ODE (Eq. 30) was solved numerically to obtain $\hat{h}$ and $\theta_{f s}$ as functions of $\hat{r}$ throughout all of region III. In this region, the initial conditions were $\hat{h}\left(\hat{r}=\hat{r}_{1}\right)=\hat{h}_{1}$ and $\theta_{f s}\left(\hat{r}=\hat{r}_{1}\right)=1$.

Non-dimensionalizing Eq. 28 and Eq. 29 results in expressions for $\mathrm{Nu}$ and $\Delta \widehat{T}$ as functions of $\hat{r}$ in this final region.

$$
N u=\frac{3 \pi \theta_{f s}}{2 \hat{h}+3 \hat{\lambda}_{T}}
$$

$$
\Delta \widehat{T}=\frac{3 \widehat{\lambda}_{T} \theta_{f s}}{2 \hat{h}+3 \hat{\lambda}_{T}}
$$

The systems of equations for regions I, II, and III were solved using LSODA (a version of the Livermore Solver for Ordinary Differential equations) as implemented by the Mathematica $\AA$ solver software. The routine switches automatically between a multi-order (1-12) Adams method for non- 
stiff problems and a backward-difference method for stiff problems. The numerical error in determining the local Nusselt number is $\pm 1 \times 10^{-4}$.

\section{Results}

\subsection{Comparison with previous results}

First, we compare our solution approach to previous work of Chaudhury for jet impingement on a classical smooth no-slip surface [25]. Chaudhury showed that the Reynolds number dependence could be accounted for using the scaling shown in Fig. 2, where the product $\hat{r}^{2} \overline{N u} R e^{-1}$ is shown as a function of $\hat{r} R e^{1 / 3}$. The results of Fig. 2 correspond to the no-slip and matching temperature boundary conditions for Prandtl numbers of 2, 5, and 8. Excellent agreement exists between the present results and those of Chaudhury [25]. Small discrepancies between the models arise because the present work approximates the velocity and temperature profiles with third-order polynomials, whereas, Chaudhury used fourth-order polynomial profiles in regions I and II and a similarity solution within region III.

A similarity solution is not possible when a uniform slip velocity exists [41], which is the influence we are investigating. Additionally, Chaudhury selected fourth-order polynomials so that the thermal boundary layer thickness would match the hydrodynamic boundary layer thickness when $\operatorname{Pr}=1$. The present work builds upon that of a previous hydrodynamic model [37], which utilized third-order polynomials and obtained excellent agreement with the prior no-slip solution [19]. The approach is similar to that taken by Chaudhury in that the same order polynomials as the hydrodynamic model are selected so that the hydrodynamic and thermal boundary layers are equal 
when $\operatorname{Pr}=1$.

\subsection{Local behavior: Influence of slip velocity and temperature jump, Reynolds} number, and Prandtl number.

This section of the paper explores the influence of slip velocity and temperature jump on the local thermal boundary layer growth and temperature profiles. The thermal boundary layer thickness and film height, $\hat{\delta}_{T}$ and $\hat{h}$, are shown as functions of $\hat{r}$ for varying $\hat{\lambda}, \hat{\lambda}_{T}, R e$, and $\operatorname{Pr}$ in Fig. 3. For all scenarios considered, $\hat{\delta}_{T}$ increases (starting at 0 ) with increasing $\hat{r}$ until it eventually merges with $\hat{h}$. The $\hat{\delta}_{T}$ curves are qualitatively similar across the entire range of temperature jump lengths: the thermal boundary layer

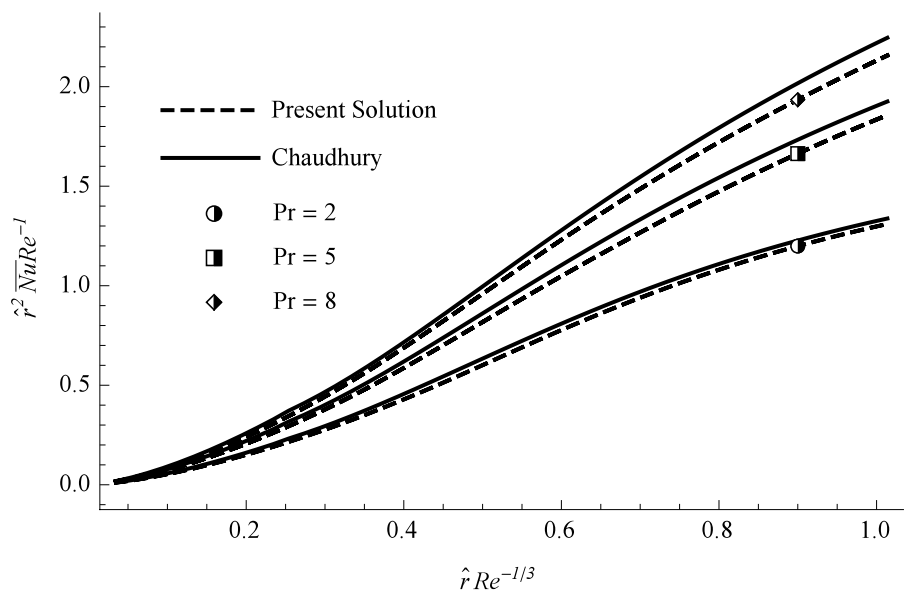

Figure 2: $\hat{r} \overline{N u} R e^{-1}$ as a function of $\hat{r} R e^{-1 / 3}$ for the classical no-slip and matching wall temperature scenario for $\mathrm{Pr}$ ranging from 2 to 8. Present solution results (dashed lines) are compared to corresponding solutions presented by Chaudhury (solid lines) [25].

grows similar to the classical behavior with the thickness initially increasing with the radial coordinate. The growth rate in $\hat{\delta}_{T}$ decreases with increasing 
radial coordinate up until the existence of an inflection point in the $\hat{\delta}_{T}$ vs. $\hat{r}$ plot, which marks the transition from region I to region II. This inflection occurs because the hydrodynamic boundary layer has merged with the film thickness in region II. The magnitude of the inflection decreases with increasing temperature jump length, increasing $R e$, and decreasing $P r$. At $\hat{r}$ values beyond the inflection point, the growth rate of $\hat{\delta}_{T}$ increases because $\hat{h}$ is decreasing less rapidly as the jet spreads into a thin film [37].

Panel (a) of Fig. 3 shows the thin film height and thermal boundary layer thickness as a function of $\hat{r}$ and for varying values of $\hat{\lambda}_{T}$ ranging from $\hat{\lambda}_{T}=0$ (the no-slip condition) to $\hat{\lambda}_{T}=0.2$. The Reynolds number and Prandtl number for these cases are constant at $R e=9,000$ and $\operatorname{Pr}=5$. The local values of both $\hat{h}$ and $\hat{\delta}_{T}$ decrease as $\hat{\lambda}$ increases. $\hat{h}$ decreases since the velocity near the wall is higher and the hydrodynamic boundary layer develops more slowly as a result of lower wall shear. The thermal boundary layer thickness develops more slowly due to increased thermal resistance at the wall caused by the temperature jump, which results in a decreased heating rate.

Panel (b) of Fig. 3 highlights the influence of varying Re while the temperature jump length is kept constant at $\hat{\lambda}_{T}=0.1$. The Reynolds number is varied from 3,000 to 15,000 and, for all cases, $\operatorname{Pr}=5$. Increasing $R e$ results in an increase in the momentum of the thin film, thus decreasing the thickness of $\hat{\delta}, \hat{\delta}_{T}$, and $\hat{h}$. Similar to previous work regarding slip length [37], increasing $\hat{\lambda}$ has a similar effect as increasing $R e$ which is obvious when comparing the results of panel (a) and panel (b). 


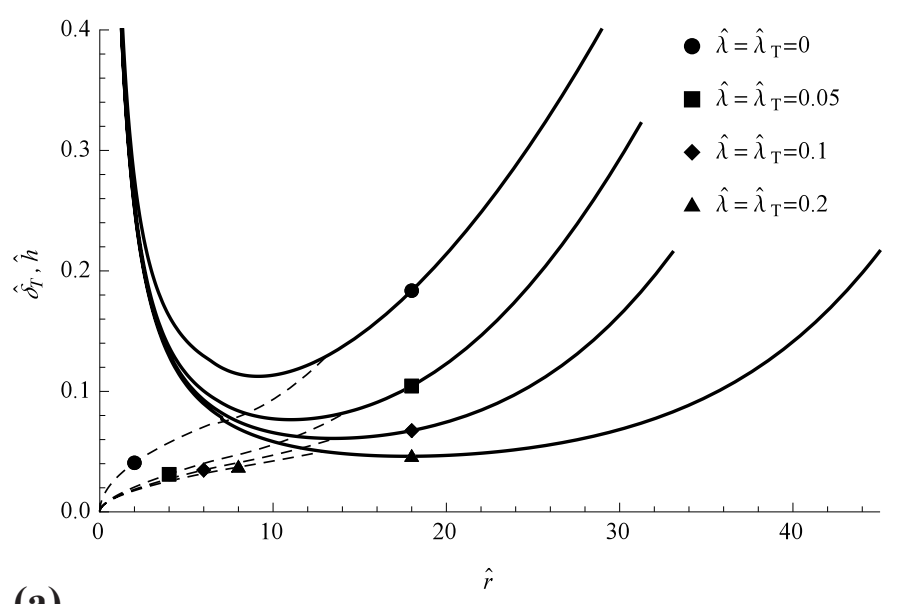

(a)

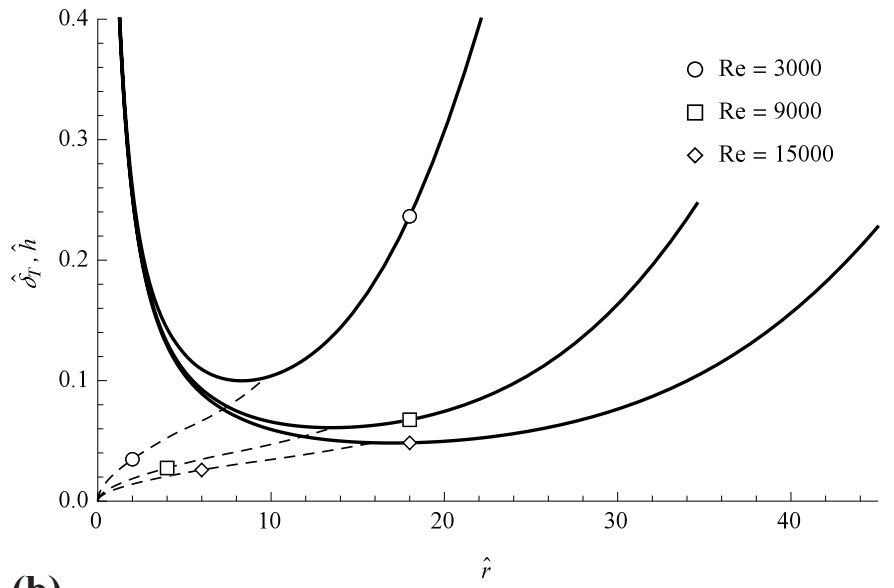

(b)

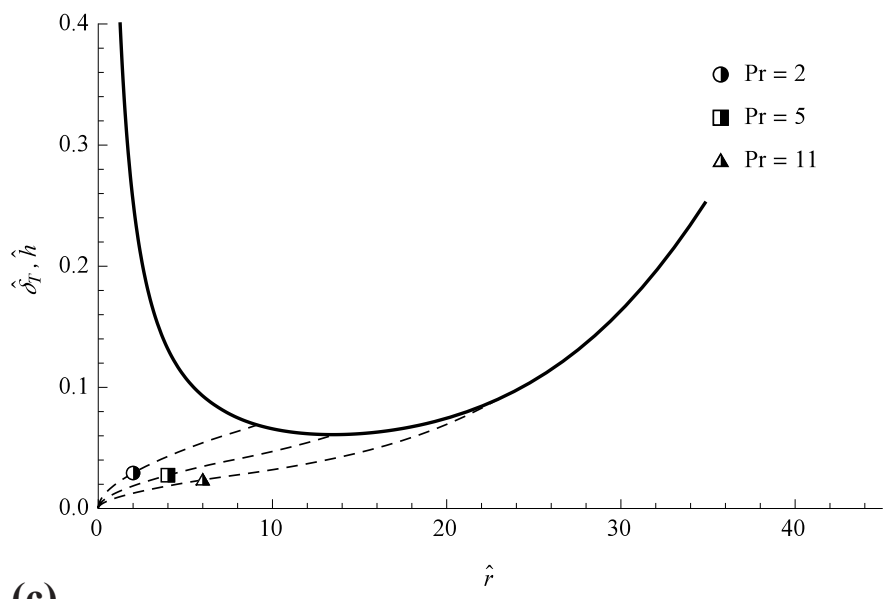

(c)

Figure 3: Film thickness and thermal boundary layer thickness as a function of radial position. Panel (a): $\operatorname{Pr}=5, R e=9,000$, and $\hat{\lambda}$ and $\hat{\lambda}_{T}$ varying from 0 to 0.2. Panel (b): $\operatorname{Pr}=5, \hat{\lambda}=\hat{\lambda}_{T}=0.1$, and $R e$ varying from 3,000 to 15,000 . Panel (c): $R e=9,000$, $\hat{\lambda}=\hat{\lambda}_{T}=0.1$, and $\operatorname{Pr}$ varying from 2 to 11 . 
The influence of variations in $\operatorname{Pr}$ is illustrated in panel (c) for $\hat{\lambda}_{T}=0.1$ and $R e=9,000$. Of course, the hydrodynamic solution is not affected by varying $\operatorname{Pr}$ and increasing $\operatorname{Pr}$ expectedly causes the thermal boundary layer to grow more slowly with $\hat{r}$ as the thermal energy diffuses more slowly.

Non-dimensional temperature profiles $(\theta(\hat{z}))$ at two different normalized radial locations, $\hat{r}=10$ and $\hat{r}=30$, are shown in Fig. 4 for various combinations of $R e, P r$, and $\hat{\lambda}_{T}$. The left column (panels (a), (c), and (e)) show profiles evaluated at $\hat{r}=10$ and the right column (panels (b), (d), and (f)) show profiles evaluated at $\hat{r}=30$. The vertical extent of the profiles vary because all profiles terminate at the height of the thin film, $\hat{h}$, which varies with $\hat{r}, R e$, and $\hat{\lambda}$, as shown in Fig. 3. At $\hat{h}$, the temperature gradient is zero as required by the adiabatic boundary condition at the free surface. Also, due to the non-dimensionalization $\left(\theta=\left(T-T_{w}\right) /\left(T_{j}-T_{w}\right)\right), \theta=1$ when $T=T_{j}$ and $\theta=0$ when $T=T_{w}$. For all scenarios, as $\hat{z}$ increases (moving from the surface), $\theta$ increases, concomitant with a greater difference between the local temperature and the wall temperature.

In the left column of panels (a, c, e) at $\hat{r}=10$, many of the profiles are not fully developed and plateau at $\theta=1$ where $T=T_{j}$. This occurs because this portion of the thin film has not yet been influenced by the conditions at the wall. For the temperature profiles evaluated at $\hat{r}=30$, the profile is located within region III where the thermal boundary layer is fully developed. Here a plateau does not exist and the profile stretches across the entire thin film. 


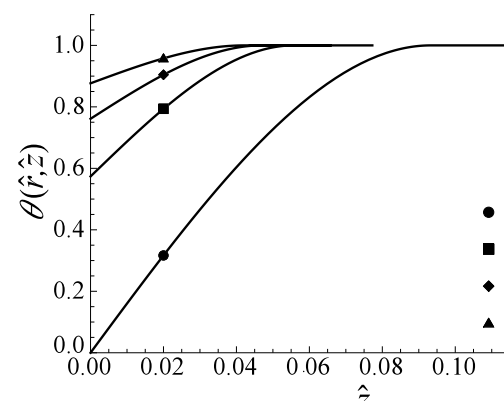

(a)

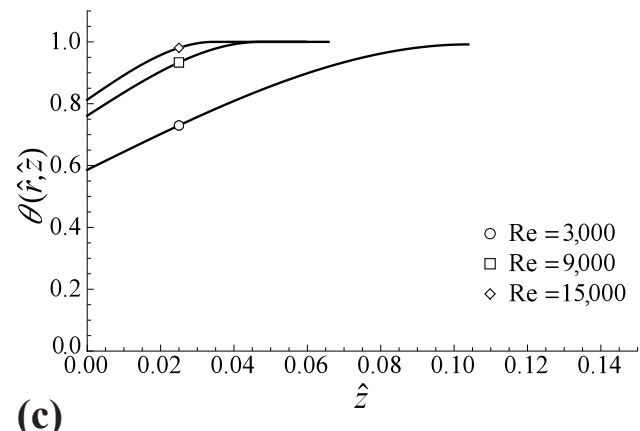

(c)

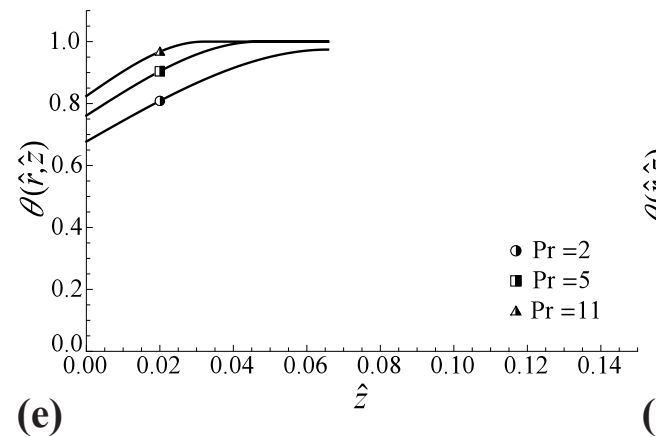

(e)

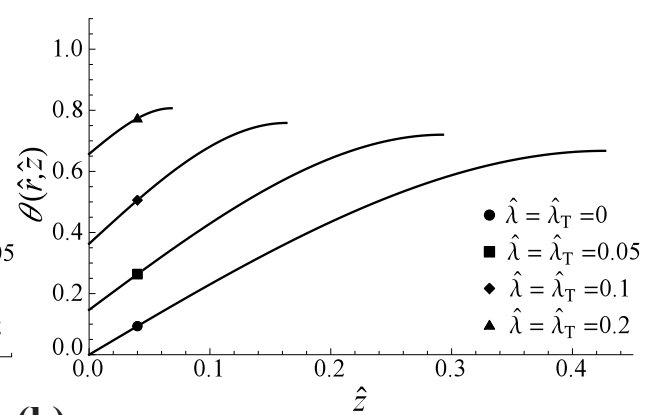

(b)

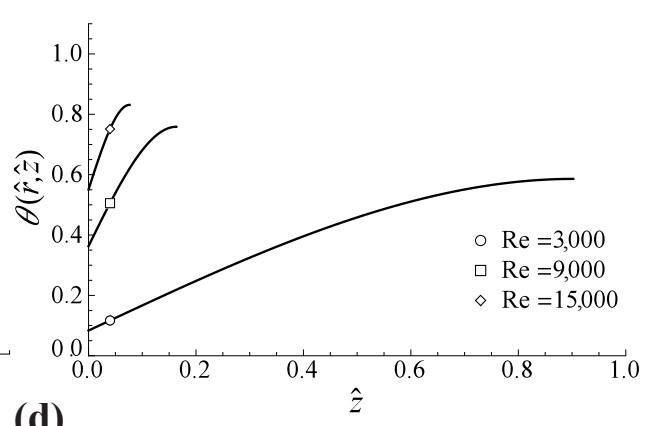

(d)

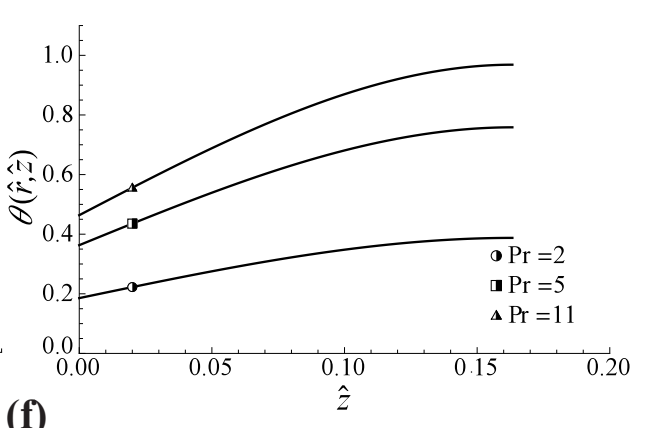

(f)

Figure 4: Non-dimensional temperature profiles, as functions of wall-normal coordinate, $\hat{z}$, at two $\hat{r}$ locations. Panels (a), (c), and (e): $\hat{r}=10$. Panels (b), (d), and (f) $: \hat{r}=30$. Panels (a) and (b): $\operatorname{Pr}=5, R e=9,000$, and $\hat{\lambda}_{T}$ ranging from 0 to 0.2. Panels (c) and (d): $\operatorname{Pr}=5, \hat{\lambda}=\hat{\lambda}_{T}=0.1$, and $\operatorname{Re}$ ranging from 3,000 to 15,000. Panels (e) and (f): $R e=9,000, \hat{\lambda}_{T}=0.1$, and $\operatorname{Pr}$ ranging from 2 to 11 . 
For all profiles shown, the temperature profiles that do not pass through the origin exhibit an apparent temperature jump at the wall due to the superhydrophobic temperature jump condition. This temperature jump may be as large as $90 \%$ of the temperature difference between the wall and the jet (see panel (a)). In panels (a) and (b) $\operatorname{Re}$ and $\operatorname{Pr}$ are held constant at 9,000 and 5 respectively and $\hat{\lambda}_{T}$ is varied through $0,0.05,0.1$, and 0.2 .

The results of panels (a) and (b) highlight the difference in thickness of the thermal boundary layer for the no-slip and no temperature jump case $\left(\hat{\lambda}=\hat{\lambda}_{T}\right)$ relative to the slip and temperature jump cases $\hat{\lambda}=\hat{\lambda}_{T}>0$. As $\hat{\lambda}_{T}$ increases, there is an increased resistance to heat flux at the surface and consequently a smaller $\hat{\delta}_{T}$.

The results of panels (c) and (d) correspond to a fixed value of $\operatorname{Pr}=5$ and $\hat{\lambda}=\hat{\lambda}_{T}=0.1$ while $R e$ is varied through the values: $3,000,9,000$, and 15,000. As Re increases, the temperature jump at the wall increases at both $\hat{r}=10$ and $\hat{r}=30$, and this is more pronounced at lower $\hat{r}$. Increasing Re has a similar effect as increasing $\hat{\lambda}_{T}$ on the temperature jump at the wall, although larger $R e$ yields larger wall-normal gradients in $\theta$ while increasing $\hat{\lambda}_{T}$ leads to smaller gradients. The results for panels (e) and (f) correspond to $R e=9,000$ and $\hat{\lambda}=\hat{\lambda}_{T}=0.1$ while $\operatorname{Pr}$ is varied through the values of 2,5 , and 11. As $\operatorname{Pr}$ increases, the thermal energy diffuses more slowly from the wall into the liquid, as is typical, and $\theta$ approaches zero more slowly with increasing $\hat{r}$. Further, the results reveal that the wall non-dimensional temperature jump also increases with increasing Prandtl number.

A local $N u$ may be obtained from the local values of $\hat{\delta}_{T}$ and $\theta_{f s}$ using Eq. 18 and 31. Shown in Figs. 5 and 6 is the local Nusselt number as a 
function of $\hat{r}$ and the results demonstrate the influence exerted by variations in slip length and temperature jump length. For all scenarios (all values of $\left.\hat{\lambda}_{T}\right), N u$ decreases with increasing $\hat{r}$ as expected. The transition from region I to II for all scenarios is indicated by a plus sign + and the region II to III transition is indicated by an X.

Results are shown for $R e=3,000$ (panel(a)) and 15,000 (panel (b)) at constant $\operatorname{Pr}=5$ in Fig. 5 for $\hat{\lambda}_{T}=0,0.05,0.1$, and 0.2. Results are shown for the same values of $\hat{\lambda}_{T}$ in Fig. 6, but for $\operatorname{Pr}=2,5$, and 11 and at a constant $R e=9,000$. $N u$ increases (at fixed $\hat{r}$ ) with increasing $R e$ or $\operatorname{Pr}$ for the no-slip and matching temperature cases as expected. The influence of $R e$ or $\operatorname{Pr}$ decreases in cases with a non-zero temperature jump length. The negligible change in $N u$ with $R e$ or $\operatorname{Pr}$ at the stagnation point $(\hat{r}=0)$, for cases with a non-zero temperature jump length, illustrates this effect. In general, these surfaces have smaller and more uniform $N u$ and these effects increase as the temperature jump length increases.

We would like to further emphasize the departure from the smooth surface behavior $\left(\hat{\lambda}_{T}=0\right)$ to what prevails for the superhydrophobic scenarios $\left(\hat{\lambda}_{T} \neq 0\right)$. We first consider the transport near the jet centerline and stagnation region $(\hat{r}<15)$, where the influence of temperature jump exerts the greatest influence and large variations in $N u$ exist for the range of $\hat{\lambda}_{T}$ values considered. For all scenarios, increases in $\hat{\lambda}_{T}$ yield notably smaller values of $N u$, compared to the smooth surface case. For example, for the $R e=15,000$, $\operatorname{Pr}=5$ scenario and at $\hat{r}=4, N u$ decreases by nominally $60 \%, 77 \%$, and $90 \%$ for the $\hat{\lambda}_{T}=0.05,0.1$, and 0.2 scenarios, respectively. The dependence on $\hat{\lambda}_{T}$ is greatest at the jet centerline and decreases moving radially outward. 


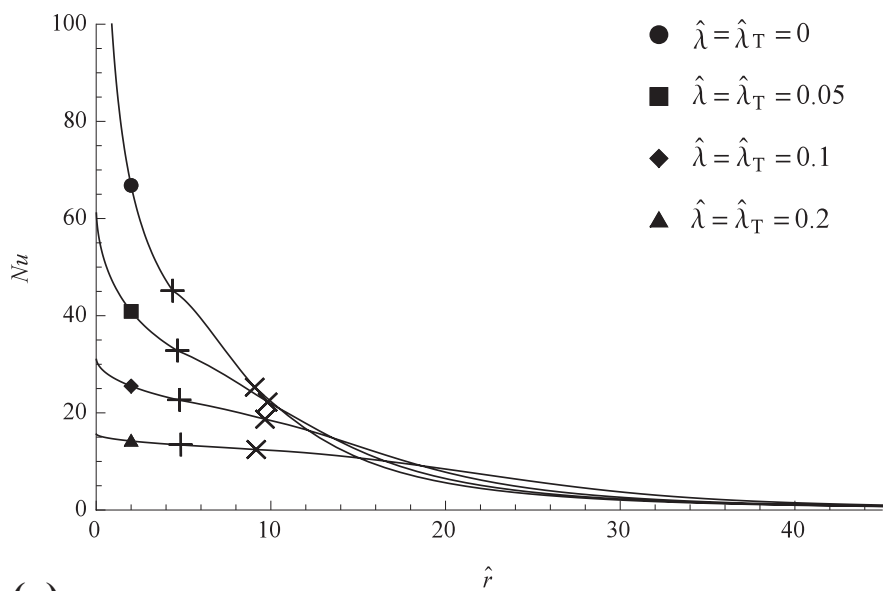

$$
R e=3,000
$$

(a)

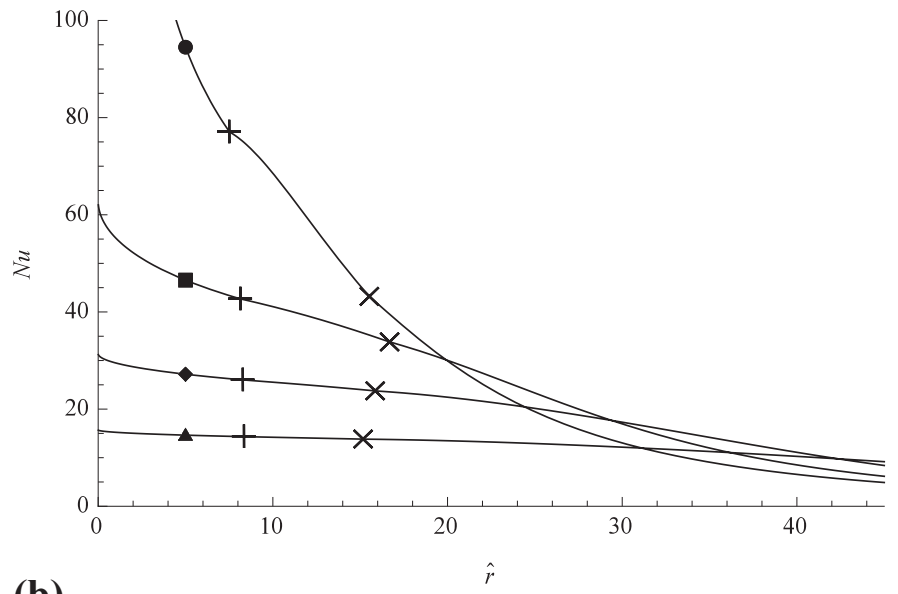

(b)

$\operatorname{Re}=15,000$

Figure 5: $N u$ as function of $\hat{r}$ for $\hat{\lambda}_{T}$ ranging from 0 to 0.2. $P r=5$. Panel (a): $R e=3,000$. Panel (b): $R e=15,000$. A $R e=9,000$ and $\operatorname{Pr}=5$ case is not shown here but is included in Fig. 10. Plus signs + indicate the region I to region II transition and Xs indicate the region II to region III transition. 


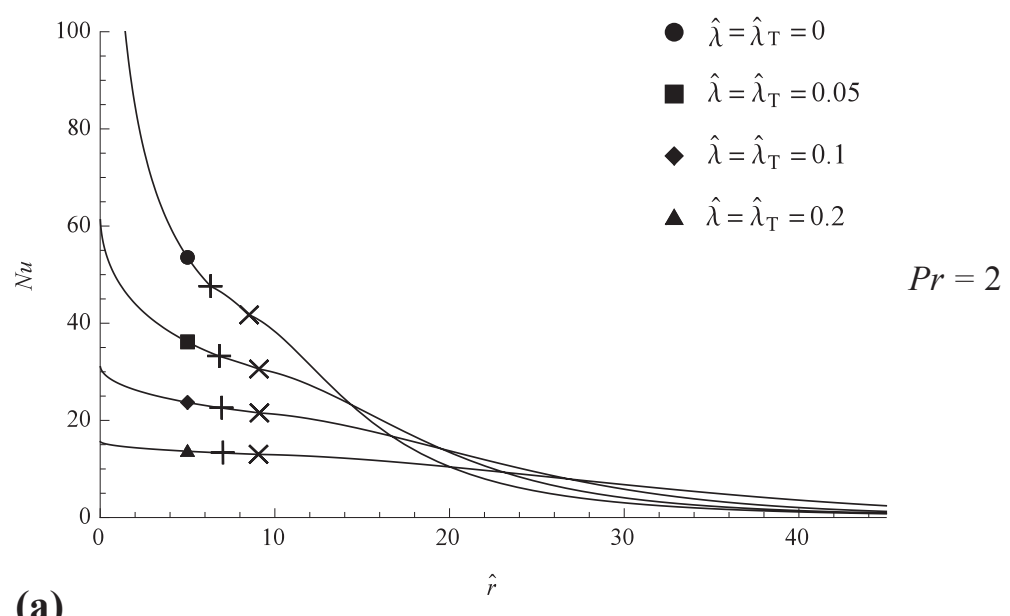

(a)

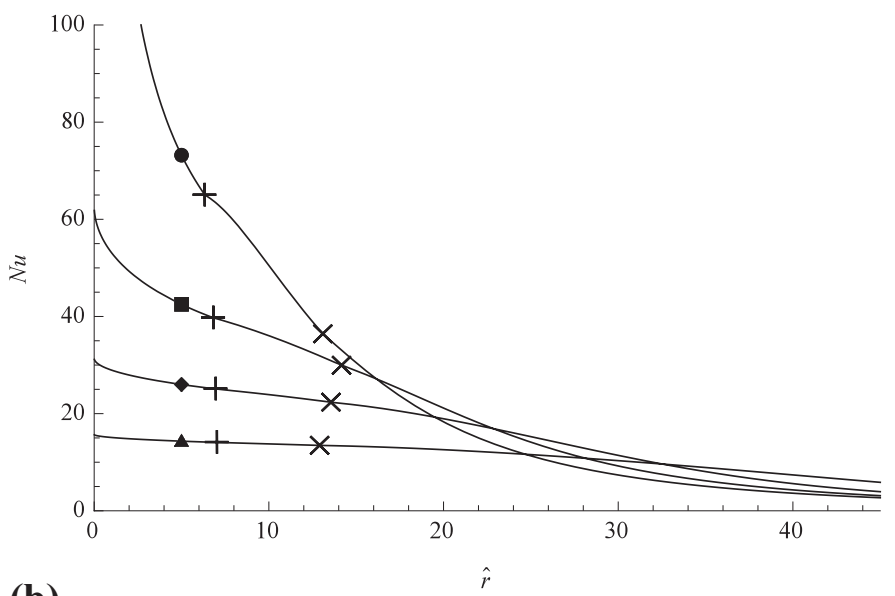

(b)

$$
\operatorname{Pr}=5
$$

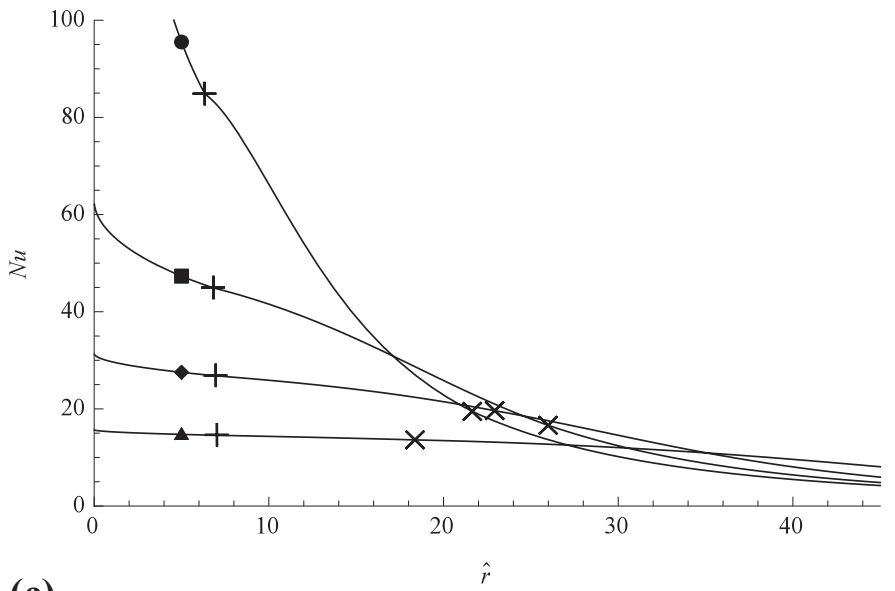

(c)

$\operatorname{Pr}=11$

Figure 6: $N u$ as function of $\hat{r}$ for $\hat{\lambda}_{T}$ ranging from 0 to 0.2. $R e=9,000 . \operatorname{Pr}$ is varied through 2, 5, and 11 in panels (a), (b), and (c), respectively. As in Fig. 5, plus signs + indicate the region I to region II transition and Xs indicate the region II to region III transition. 
The relative (compared to the smooth surface case) decrease in magnitude of $N u$ with increasing $\hat{\lambda}_{T}$ is a strong function of $R e$ and a moderate function of $P r$. In general, the relative decrease in $N u$ (with increasing $\hat{\lambda}_{T}$ ) is larger as either $\operatorname{Re}$ or $\operatorname{Pr}$ are increased, as illustrated by the data in both figures.

Two other points deserve discussion relative to the data of Figs. 5 and 6. First, as $\hat{\lambda}_{T}$ increases to 0.2 , the overall variation with $N u$ varies from approximately 18 at the stagnation point to approximately 14 at $\hat{r}=30$, in stark contrast to the variation observed in the $\hat{\lambda}_{T}=0$ scenario. The implication of this behavior is that the local heat flux becomes much more uniform for the larger temperature jump length scenarios. Indeed, at the larger $R e$, $\operatorname{Pr}$, and $\hat{\lambda}_{T}$ explored, $N u$ is approaching a uniform value for all $\hat{r}$, an interesting and unexpected result. The second point is related to the first. At sufficiently large values of $\hat{r}$ (the exact point is dependent on $R e$ and $\operatorname{Pr}$ but is generally between 15 and 25 radii from the stagnation point), the magnitude of $N u$ for the $\hat{\lambda} \neq 0$ scenarios exceeds the value for the $\hat{\lambda}=0$ surface. The implication here is that the heat transfer is actually increased on the superhydrophobic surfaces at large radial locations. This behavior prevails because of the dramatic decrease in heat transfer in the stagnation region, which results in a smaller temperature increase in the liquid temperature in the liquid film. Consequently, at larger radii, the driving temperature difference between the wall and the mean liquid temperature is greater relative to the smooth surface case and results in modestly higher local heat transfer rates. Further, it is important to point out that even small values of $\hat{\lambda}_{T}$ (see $\hat{\lambda}_{T}=0.05$ data) result in significant departures in the local $N u$ magnitude (relative to the smooth surface) and may be important when considering 
data derived from classical jet impingement experiments on reported smooth surfaces.

For small temperature jump length, the effect of increasing either Re or $\operatorname{Pr}$, causes the local Nusselt number to increase (seen both in Figs. 5 and 6). However, as the slip length and the jump length increase, changing $\operatorname{Re}$ or $\mathrm{Pr}$ has less effect. Consider the $\hat{\lambda}_{T}=0.2$ case in Figs. 5 and 6 for which there is little apparent change. Here heat transfer is determined by the thermal resistance at the wall instead of the flow conditions.

Shown in Fig. 7 is the average Nusselt number, $\overline{N u}$, for smooth surfaces $\left(\hat{\lambda}_{T}=0\right)$ as a function of $\hat{r}$ and the results demonstrate the influence exerted by variations in $R e$ and $\operatorname{Pr}$. Figure 7 provides a point of comparison for superhydrophobic scenarios $\left(\hat{\lambda}_{T} \neq 0\right)$ which are discussed further below. Results are shown for $R e=3,000,9,000$, and 15,000 at a constant $\operatorname{Pr}=5$ (panel (a)) and for $\operatorname{Pr}=2,5$, and 11 at a constant $R e=9,000$ (panel (b)). In general, $\overline{N u}$ decreases with increasing $\hat{r}, R e$, and $\operatorname{Pr}$ in agreement with well-known behavior.

A fractional reduction in the average thermal transport, $1-\overline{N u} / \overline{N u}_{0}$, is used to quantify the reduction in $\overline{N u}$ for the SHPo cases, where $\overline{N u}_{0}$ is $\overline{N u}$ at $\hat{\lambda}_{T}=0$. A fractional reduction of 1 indicates that the thermal transport has decreased by $100 \%$. Shown in Fig. 8 is $1-\overline{N u} / \overline{N u}_{0}$ as a function of $\hat{r}$ and the results demonstrate the influence exerted by variations in the temperature jump length. Results are shown for $R e=3,000$ and 15,000 (panel (a)) at constant $\operatorname{Pr}=5$ with $\hat{\lambda}_{T}=0.1$ and 0.2 and for $\operatorname{Pr}=2,5$, and 11 (panel(b)) at constant $R e=9,000$ with $\hat{\lambda}_{T}=0.1$ and 0.2 . In general, the fractional reduction decreases with increasing $\hat{r}$. This is expected in agreement with 


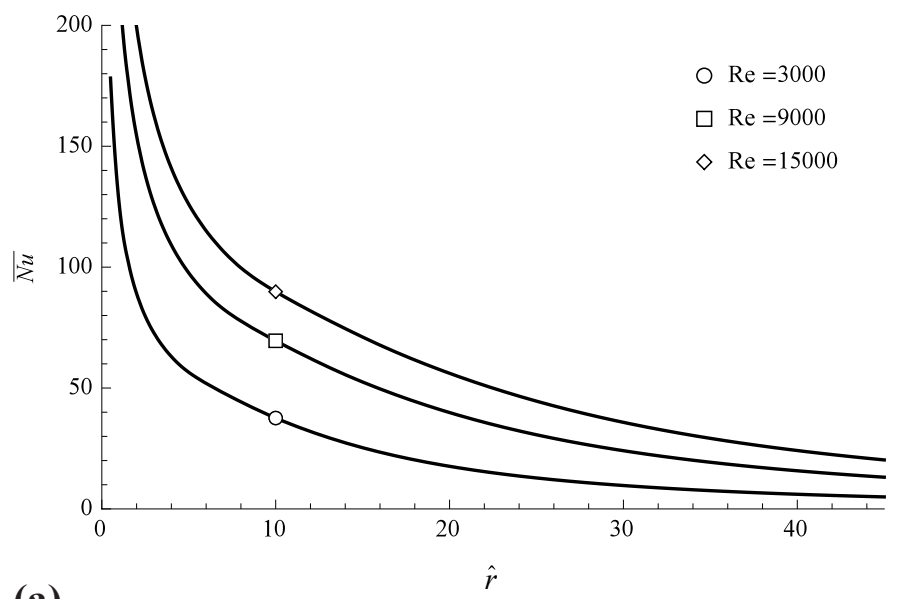

(a)

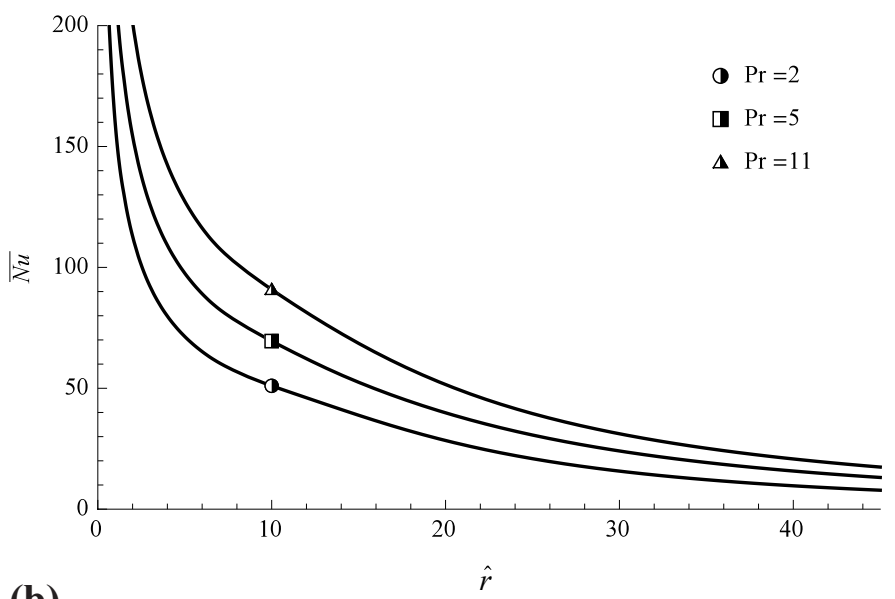

(b)

Figure 7: $\overline{N u}$ for no-slip and matching temperature surface as a function of the radius, $\hat{r}$, of the disk over which $N u$ is averaged. Panel (a): $\operatorname{Pr}=5$. Re is varied from 3,000 to 15,000. Panel (b): $\operatorname{Re}=9,000 . \operatorname{Pr}$ is varied from 2 to 11 . 


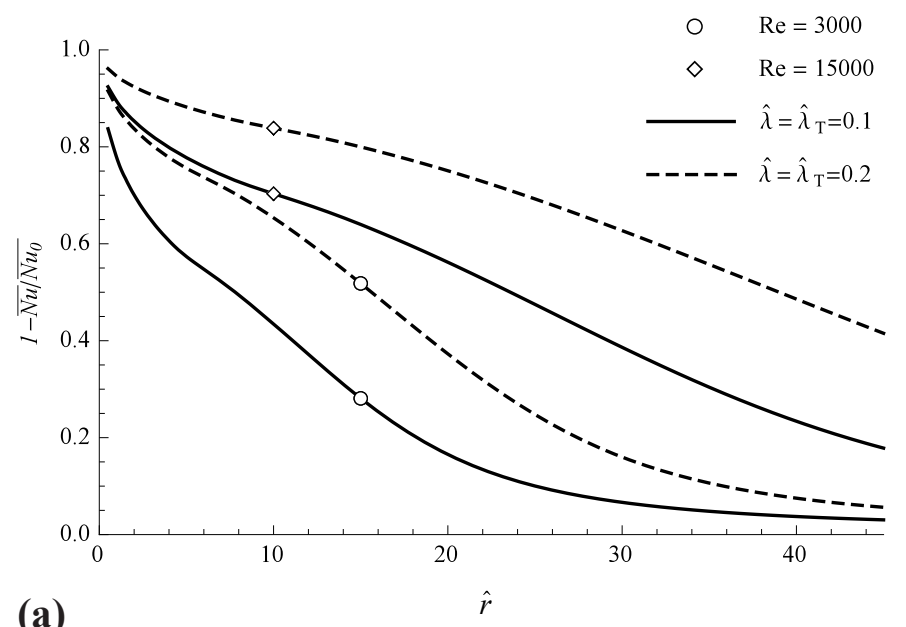

(a)

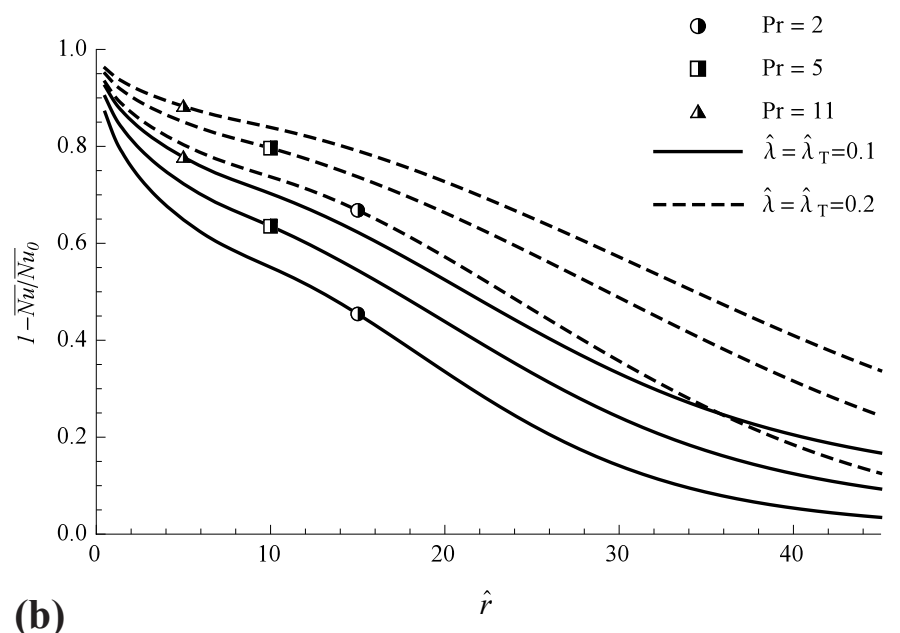

(b)

Figure 8: Fractional reduction in Nusselt number, $1-\overline{N u} / \overline{N u}_{0}$, as a function of $\hat{r}$ over which $N u$ is averaged. $\overline{N u}_{0}$ is the average Nusselt number for the no-slip and matching temperature condition. $\hat{\lambda}_{T}=0.1$ is indicated by a solid line, while $\hat{\lambda}_{T}=0.2$ is indicated by a dashed line. Panel (a): $\operatorname{Pr}=5$ and $R e$ equals 3,000 and 15,000. Panel (b): $\operatorname{Re}=$ 9,000. $\operatorname{Pr}$ equals 2, 5, and 11 . 
the same reasoning provided previously for the influence of $\hat{r}$ on $\overline{N u}$. Increasing $\hat{\lambda}_{T}$ yields increased reduction in $\overline{N u}$, as expected from the results for $N u$

Consider the disk radius $\hat{r}=10$ position in panels (a) and (b). For the $\hat{\lambda}_{T}=0.1$ case, the fractional reduction increases from nominally 0.45 to 0.65 when Re increases from 3,000 to 15,000. Further, the fractional reduction increases through nominally $0.55,0.65$, and 0.7 as $\operatorname{Pr}$ increases through 2, 5, and 11. We emphasize this unexpected result that increasing $\operatorname{Re}$ or $\operatorname{Pr}$ on a SHPo surface increases the fractional reduction in $\overline{N u}$ at a fixed $\hat{\lambda}_{T}$ value. This behavior demonstrates coupled interactions between $\hat{\lambda}_{T}$ and $R e$ and $\operatorname{Pr}$ on the magnitude of $\overline{N u}$.

The actual Nusselt numbers may be obtained by using the value of the fractional reduction displayed in this figure in conjunction with $\overline{N u}$ for the smooth surface plotted in Fig. 7 or, for select radii, from Figs. 9 and 10.

To further quantify the influence of $\hat{\lambda}_{T}$ on $\overline{N u}$, the average Nusselt number is shown as a function of $\hat{\lambda}_{T}$ at specific $\hat{r}$ values $(10,20,30,40)$ in Fig. 9. For all scenarios (all values of $\hat{r}$ explored), $\overline{N u}$ decreases with increasing $\hat{\lambda}_{T}$ as expected. Results are shown at $\operatorname{Pr}=5$ and $R e=3,000$ (panel(a)) and for the same values of $\operatorname{Pr}$ but at $R e=15,000$ (panel (b)). Increasing $\hat{\lambda}_{T}$ reduces thermal transport dramatically when $N u$ is averaged over the region near the stagnation point. A $66 \%$ reduction is observed in panel (b) at $\hat{r}=$ 10 when $\hat{\lambda}_{T}$ increases from 0 to 0.1 . Less reduction is observed for larger $\hat{r}$ values. Consider a disk of radius $\hat{r}=40$ where a $25 \%$ decrease is observed as $\hat{\lambda}_{T}$ increases from 0 to 0.2 . Similar behavior was observed across the entire range of $\operatorname{Re}$ and $\operatorname{Pr}$ considered. 


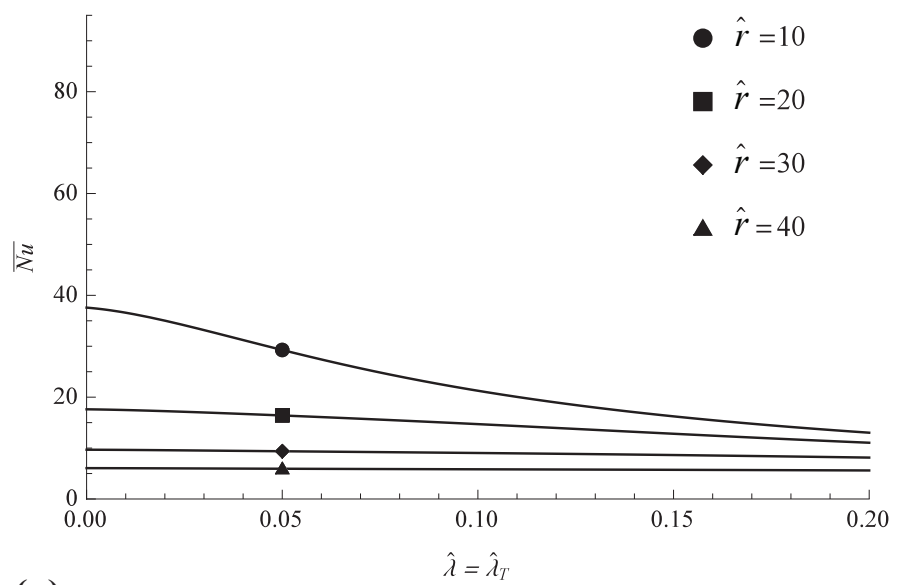

(a)

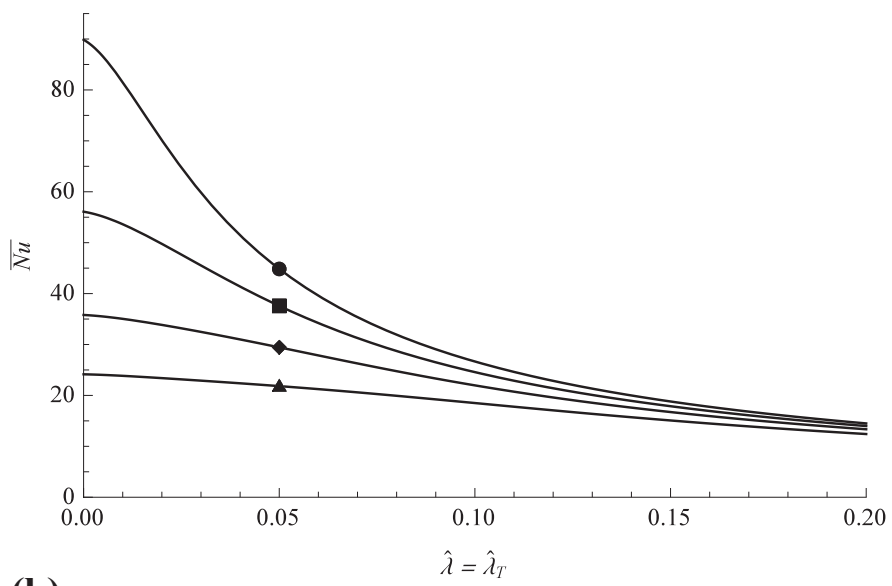

(b)

Figure 9: $\overline{N u}$ as a function of $\hat{\lambda}_{T}$ for range of $\hat{r}$, from 10 to 40. $\operatorname{Pr}=5$. Panel (a): $R e=3,000$. Panel (b): $R e=15,000$. 
Shown in Fig. 10 is the average Nusselt number at a fixed $\hat{r}$ value of 15 as a function of $\hat{\lambda}_{T}$. These results demonstrate the influence exerted by variations in $\operatorname{Re}$ (panel a) and $\operatorname{Pr}$ (panel b) for $\operatorname{SHPo}\left(\hat{\lambda}_{T} \neq 0\right)$ surfaces. For all scenarios (all values of $R e$ and $\operatorname{Pr}$ explored), $\overline{N u}$ decreases as expected with increasing $\hat{\lambda}_{T}$. Results are shown for $R e=3,000,7,000,11,000$, and 15,000 at constant $\operatorname{Pr}=5$ (panel (a)) and for $\operatorname{Pr}=2,5,8$, and 11 at constant $R e=7,000$ (panel (b)). For all cases, $\hat{r}=15$. Results are shown for the same range of $\hat{\lambda}_{T}$ (0 to 0.2$)$ in both panels. For smooth surfaces, it is well known that $\overline{N u}$ increases with $R e$ and $P r$. We observe this trend for all $\hat{\lambda}_{T}$ in panels (a) and (b), respectively.

We emphasize the surprising interaction of varying $R e$ and $\operatorname{Pr}$ with varying $\hat{\lambda}_{T}$. Consider the case of $\hat{\lambda}_{T}=0.01$ in panels (a) and (b). In panel (a) between $R e=3,000$ and $R e=15,000, \overline{N u}$ increases by $164 \%$. In panel (b) between $\operatorname{Pr}=2$ and $\operatorname{Pr}=11, \overline{N u}$ increases by $75 \%$. In contrast, compare the increase at $\hat{\lambda}_{T}=0.2$. There the increase is nominally $20 \%$ for both $R e$ (increasing from 3,000 to 15,000) and $\operatorname{Pr}$ (increasing from 2 to 11). At small $\hat{\lambda}_{T}$, varying $\operatorname{Re}$ or $\operatorname{Pr}$ alters $\overline{N u}$ dramatically while at large $\hat{\lambda}_{T}$ variations in $R e$ and $\operatorname{Pr}$ exercise little influence on $\overline{N u}$ and the $\overline{N u}$ vs. $\hat{\lambda}_{T}$ curves collapse together. This occurs because thermal resistance due to the surface condition becomes more significant than thermal resistance due to the convection boundary condition as $\hat{\lambda}_{T}$ increases. 


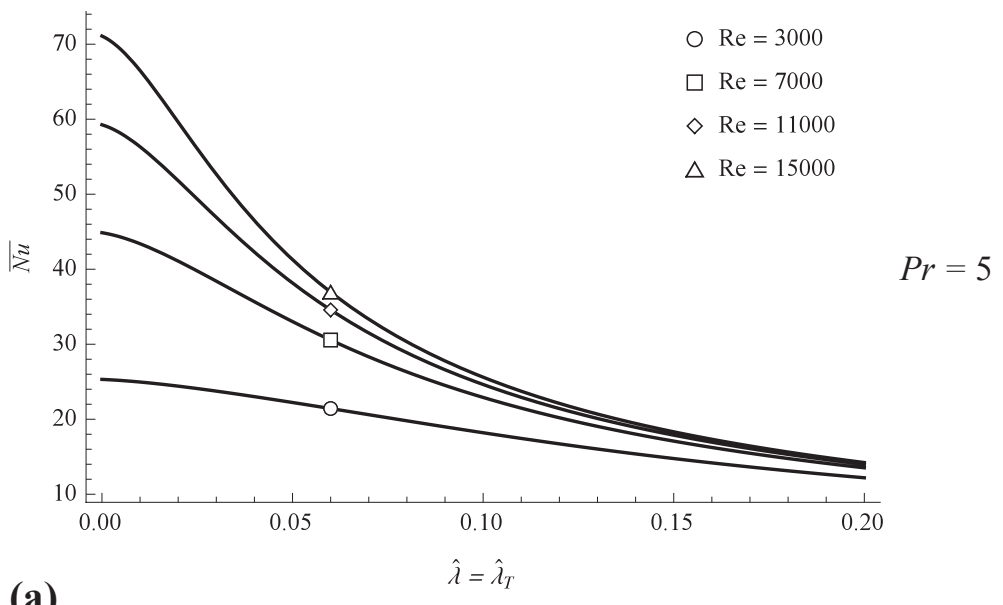

(a)

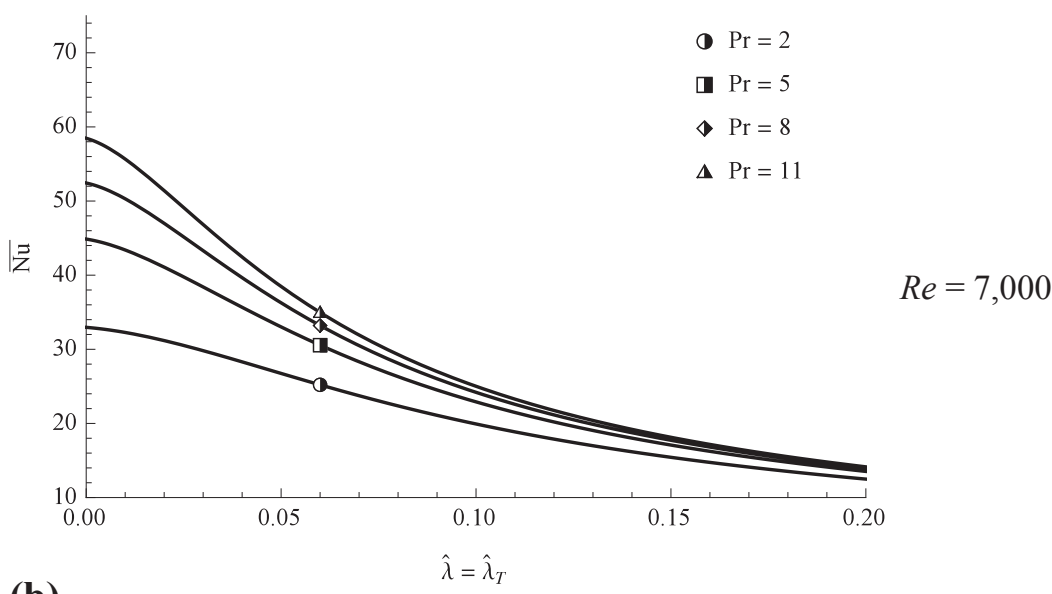

(b)

Figure 10: $\overline{N u}$ as a function of $\hat{\lambda}_{T} \cdot \hat{r}=15$. Panel (a): $\operatorname{Pr}=5$. Re varies from 3,000 to 15,000. Panel (b): $R e=7,000$. $\operatorname{Pr}$ varies from 2 through 11. 


\subsection{Relative importance of slip length and temperature jump length on the} solution

This section of the paper explores the relative importance of slip velocity compared to temperature jump on the overall transport. Shown in Fig. 11 is the average Nusselt number as a function of $\hat{\lambda}$ and $\hat{\lambda}_{T}$ at $\hat{r}=15$.

Results are shown at constant $\operatorname{Pr}=5$ and $R e=7,000$ for three cases. First, $\hat{\lambda}$ is varied from 0 to 0.2 while $\hat{\lambda}_{T}$ is held constant at $\hat{\lambda}_{T}=0$. These results are indicated by the dash-dotted line. Second, $\hat{\lambda}_{T}$ is varied from 0 to 0.2 , at a constant value of $\hat{\lambda}=0$ and these results are indicated by the dashed line. Third, $\hat{\lambda}=\hat{\lambda}_{T}$ and these results are indicated by the solid line.

For case 1 , increasing $\hat{\lambda}\left(\hat{\lambda}_{T}=0\right)$ yields an increase in $\overline{N u}$ because the wall slip velocity increases with $\hat{\lambda}$. This enhanced near-wall advection thus results in a larger $\overline{N u}$. As $\hat{\lambda}$ increases from 0 to $0.2, \overline{N u}$ increases by nominally $100 \%$. For case 2 , increasing $\hat{\lambda}_{T}(\hat{\lambda}=0)$ yields an increase in the wall thermal resistance and yields a $67 \%$ decrease in $\overline{N u}$ as $\hat{\lambda}_{T}$ increases from 0 to 0.2 . For case $3, \overline{N u}$ deviates only modestly from the solution where $\hat{\lambda}_{T}$ was varied at a constant $\hat{\lambda}=0$ (dashed curve).

These results indicate that $\hat{\lambda}_{T}$ has a much greater influence on $\overline{N u}$ than a simultaneous change in $\hat{\lambda}$ and that the increased thermal resistance caused by $\hat{\lambda}_{T}$ has a larger impact on the thermal transport than the enhanced near-wall advection due to the increased wall slip velocity. 


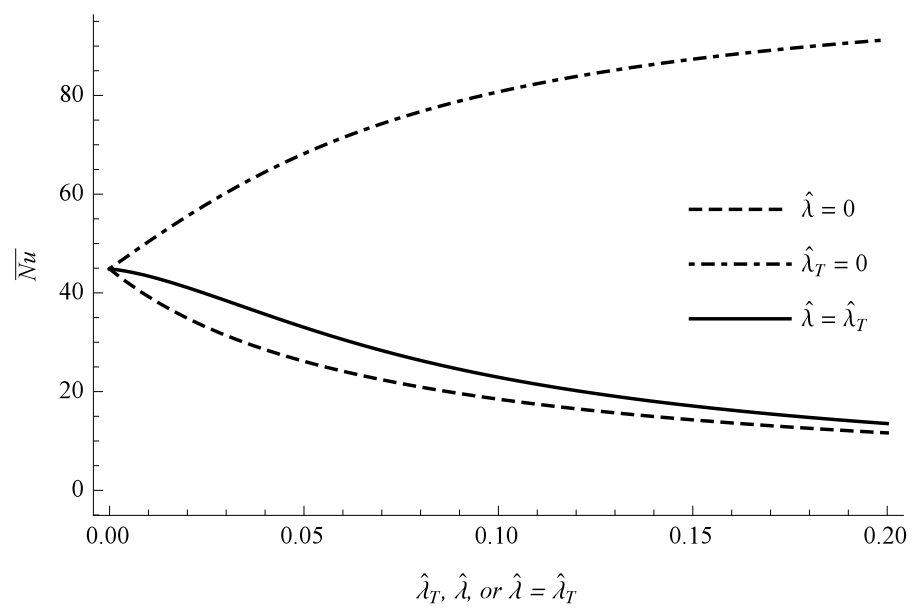

Figure 11: $\overline{N u}$ as a function of $\hat{\lambda}_{T}$ with $\hat{\lambda}=0$, as a function of $\hat{\lambda}$ with $\hat{\lambda}_{T}=0$, and as a function of $\hat{\lambda}=\hat{\lambda}_{T}$. Re $=7,000$ and $\operatorname{Pr}=5$. Nu averaged over 15 jet radii.

\section{Conclusions}

SHPo surfaces exhibit an apparent wall slip velocity and temperature jump. Although the effects of wall slip have been quantified for classical flows, interest in the influence of SHPo surfaces on thermal transport has recently emerged. A model has been developed to predict thermal transport for an axisymmetric, laminar jet impinging on a SHPo surface with isotropic slip length and temperature jump length. An integral approach was applied to solve the mass, momentum, and energy equations within the thin film of a spreading impinged jet. Third-order velocity and temperature profiles were assumed.

The results quantify local and average Nusselt numbers on heated superhydrophobic surfaces over a range of temperature jump lengths ranging from 
0 to 0.2 , jet Reynolds numbers varying from 3,000 to 15,000, and Prandtl numbers ranging from 2 to 11 .

The introduction of an isotropic SHPo surface has the following effects on thermal transport:

- The local thermal boundary layer thickness decreases relative to the classical scenario.

- Thermal transport is reduced dramatically in the vicinity of the stagnation point and this decrease becomes more significant as the level of superhydrophobicity increases.

- The Nusselt number is sensitive to the introduction of even a small temperature jump length and decreases dramatically.

- The local and average Nusselt numbers become less dependent on $R e$ and $\operatorname{Pr}$ with increasing temperature jump length.

- Average Nusselt numbers are observed to decrease by up to $60-80 \%$ for realizable SHPo surfaces and flow regimes, relative to smooth surfaces.

Additionally,

- The overall thermal transport is a much more dependent on the temperature jump length than the hydrodynamic slip length.

\section{Acknowledgments}

Funding: This work was supported by the National Science Foundation [grant number CBET-1235881]. 


\section{References}

[1] J. Ou, J. P. Rothstein, Direct velocity measurements of the flow past drag-reducing ultrahydrophobic surfaces, Physics of Fluids 17 (10) (2005) 103606. doi:10.1063/1.2109867.

[2] D. Maynes, K. Jeffs, B. Woolford, B. W. Webb, Laminar flow in a microchannel with hydrophobic surface patterned microribs oriented parallel to the flow direction, Physics of Fluids 19 (9) (2007) 093603. doi : 10.1063/1.2772880.

[3] B. Woolford, J. Prince, D. Maynes, B. W. Webb, Particle image velocimetry characterization of turbulent channel flow with rib patterned superhydrophobic walls, Physics of Fluids 21 (8) (2009) 085106. doi: $10.1063 / 1.3213607$.

[4] M. B. Martell, J. B. Perot, J. P. Rothstein, Direct numerical simulations of turbulent flows over superhydrophobic surfaces, Journal of Fluid Mechanics 620 (2009) 31-41. doi:10.1017/S0022112008004916.

[5] R. J. Daniello, N. E. Waterhouse, J. P. Rothstein, Drag reduction in turbulent flows over superhydrophobic surfaces, Physics of Fluids 21 (8) (2009) 085103. doi:10.1063/1.3207885.

[6] R. Fürstner, W. Barthlott, C. Neinhuis, P. Walzel, Wetting and selfcleaning properties of artificial superhydrophobic surfaces, Langmuir 21 (3) (2005) 956-961. doi:10.1021/la0401011.

[7] K. M. Wisdom, J. A. Watson, X. Qu, F. Liu, G. S. Watson, C.-H. Chen, Self-cleaning of superhydrophobic surfaces by self-propelled jumping 
condensate, Proceedings of the National Academy of Sciences 110 (20) (2013) 7992-7997. doi:10.1073/pnas.1210770110.

[8] A. Cowley, D. Maynes, J. Crockett, Effective temperature jump length and influence of axial conduction for thermal transport in superhydrophobic channels, International Journal of Heat and Mass Transfer 79 (2014) 573-583. doi:10.1016/j.ijheatmasstransfer.2014.08.033.

[9] R. Enright, M. Hodes, T. Salamon, Y. Muzychka, Isoflux Nusselt number and slip length formulae for superhydrophobic microchannels, Journal of Heat Transfer 136 (2014) 012402. doi:10.1115/1.4024837.

[10] M. Hodes, L. S. Lam, A. Cowley, R. Enright, S. Maclachlan, Effect of evaporation and condensation at menisci on apparent thermal slip, Journal of Heat Transfer 137 (7) (2015) 071502. doi:10.1115/1.4029818.

[11] D. Maynes, J. Crockett, Apparent temperature jump and thermal transport in channels with streamwise rib and cavity featured superhydrophobic walls at constant heat flux, Journal of Heat Transfer 136 (1) (2014) 011701. doi:10.1115/1.4025045.

[12] C. Y. Wang, C.-O. Ng, Natural convection in a vertical slit microchannel with superhydrophobic slip and temperature jump, Journal of Heat Transfer 136 (3) (2013) 034502. doi:10.1115/1.4025822.

[13] C.-O. Ng, C. Y. Wang, Natural convection in a vertical microannulus with superhydrophobic slip and temperature jump, Journal of Thermophysics and Heat Transfer 28 (2) (2014) 287-294. doi:10.2514/1. T4200. 
[14] D. Tam, V. von Arnim, G. H. McKinley, A. E. Hosoi, Marangoni convection in droplets on superhydrophobic surfaces, Journal of Fluid Mechanics 624 (2009) 101-123. doi:10.1017/S0022112008005053.

[15] I. U. Vakarelski, N. A. Patankar, J. O. Marston, D. Y. C. Chan, S. T. Thoroddsen, Stabilization of Leidenfrost vapour layer by textured superhydrophobic surfaces, Nature 489 (7415) (2012) 274-277. doi:10.1038/nature11418.

[16] L.-W. Fan, J.-Q. Li, D.-Y. Li, L. Zhang, Z.-T. Yu, Regulated transient pool boiling of water during quenching on nanostructured surfaces with modified wettability from superhydrophilic to superhydrophobic, International Journal of Heat and Mass Transfer 76 (2014) 81-89. doi:10.1016/j.ijheatmasstransfer.2014.04.025.

[17] R. Enright, N. Miljkovic, A. Al-Obeidi, C. V. Thompson, E. N. Wang, Condensation on superhydrophobic surfaces: The role of local energy barriers and structure length scale, Langmuir 28 (40) (2012) 1442414432. doi:10.1021/la302599n.

[18] C.-H. Chen, Q. Cai, C. Tsai, C.-L. Chen, G. Xiong, Y. Yu, Z. Ren, Dropwise condensation on superhydrophobic surfaces with two-tier roughness, Applied Physics Letters 90 (17) (2007) 173108. doi:10.1063/1. 2731434.

[19] E. J. Watson, The radial spread of a liquid jet over a horizontal plane, Journal of Fluid Mechanics 20 (3) (1964) 481-499. doi:10.1017/ S0022112064001367. 
[20] A. Craik, R. Latham, M. Fawkes, P. Gribbon, The circular hydraulic jump, Journal of Fluid Mechanics 112 (1981) 347-362. doi:10.1017/ S002211208100044X.

[21] J. Stevens, B. Webb, Measurements of the free surface flow structure under an impinging, free liquid jet, J. Heat Transfer 114 (1) (1992) 7984. doi:10.1115/1.2911271.

[22] J. W. Bush, J. M. Aristoff, The influence of surface tension on the circular hydraulic jump, Journal of Fluid Mechanics 489 (2003) 229238. doi:10.1017/S0022112003005159.

[23] X. Liu, J. H. Lienhard, J. S. Lombara, Convective heat transfer by impingement of circular liquid jets, Journal of Heat Transfer 113 (3) (1991) 571-582. doi:10.1115/1.2910604.

[24] B. Elison, B. W. Webb, Local heat transfer to impinging liquid jets in the intially laminar, transitional, and turbulent regimes, International Journal of Heat and Mass Transfer 37 (8) (1994) 1207-1216. doi:10. 1016/0017-9310(94)90206-2.

[25] Z. H. Chaudhury, Heat transfer in a radial liquid jet, Journal of Fluid Mechanics 20 (1964) 501-511. doi:10.1017/S0022112064001379.

[26] X. Liu, J. H. Lienhard, Liquid jet impingement heat transfer on a uniform flux surface, in: R. K. Shah (Ed.), Heat Transfer Phenomena in Radiation, Combustion, and Fires, Vol. 106, ASME HTD, 1989, pp. $523-530$. 
[27] X. S. Wang, Z. Dagan, L. M. Jiji, Heat transfer between a circular free impinging jet and a solid surface with non-uniform wall temperature or wall heat flux-1. Solution for the stagnation region, International Journal of Heat and Mass Transfer 32 (7) (1989) 1351-1360. doi:10. 1016/0017-9310 (89) 90034-3.

[28] X. S. Wang, Z. Dagan, L. M. Jiji, Heat transfer between a circular free impinging jet and a solid surface with non-uniform wall temperature or wall heat flux - 2. Solution for the boundary layer region, International Journal of Heat and Mass Transfer 32 (7) (1989) 1361-1371. doi:10. 1016/0017-9310 (89) 90035-5.

[29] J. Sung, H. Choi, J. Y. Yoo, Finite element simulation of thin liquid film flow and heat transfer including a hydraulic jump, International Journal for Numerical Methods in Engineering 46 (1) (1999) 83-101. doi:10.1002/(SICI) 1097-0207 (19990910) 46:1<83: : AID-NME665>3.0.CD;2-D.

[30] J.-J. Shu, G. Wilks, Heat transfer in the flow of a cold, two-dimensional vertical liquid jet against a hot, horizontal plate, International Journal of Heat and Mass Transfer 39 (16) (1996) 3367-3379. doi:10.1016/ 0017-9310(96)00020-8.

[31] H. Fujimoto, N. Hatta, R. Viskanta, Numerical simulation of convective heat transfer to a radial free surface jet impinging on a hot solid, Heat and Mass Transfer 35 (1999) 266-272. doi:10.1007/s002310050323.

[32] H. Fujimoto, K. Takuda, N. Hatta, Numerical simulation of transient 
cooling of a hot solid by an impinging free surface jet, Numerical Heat Transfer, Part A: Applications 36 (8) (1999) 767-780. doi:10.1080/ 104077899274444.

[33] J.-J. Shu, G. Wilks, Heat transfer in the flow of a cold axisymmetric vertical liquid jet against a hot horizontal plate, Journal of Heat Transfer 130 (1) (2008) 012202. doi:10.1115/1.2780180.

[34] V. E. Nakoryakov, B. G. Pokusaev, E. N. Toyan, Impingement of an axisymmetric liquid jet on a barrier, International Journal of Heat and Mass Transfer 21 (9) (1978) 1175-1184. doi:10.1016/0017-9310(78) 90136-9.

[35] J. Stevens, B. W. Webb, Local heat transfer coefficients under an axisymmetric, single-phase liquid jet, Journal of Heat Transfer 113 (1) (1991) 71-78. doi:10.1115/1.2910554.

[36] E. Lauga, H. Stone, Effective slip in pressure-driven Stokes flow, Journal of Fluid Mechanics 489 (2003) 55-77. doi:10.1017/ S0022112003004695.

[37] J. F. Prince, D. Maynes, J. Crockett, Analysis of laminar jet impingement and hydraulic jump on a horizontal surface with slip, Physics of Fluids 24 (9) (2012) 102103. doi:10.1063/1.4757659.

[38] J. F. Prince, D. Maynes, J. Crockett, Jet impingement and the hydraulic jump on horizontal surfaces with anisotropic slip, Physics of Fluids 26 (4) (2014) 042104. doi:10.1063/1.4870650. 
745

[39] M. G. Johnson, Liquid jet impingement experiments on micro rib and cavity patterned superhydrophobic surfaces in both Cassie and Wenzel states, Master's thesis, Brigham Young University (2012).

URL http://scholarsarchive. byu.edu/etd/3758

[40] C.-O. Ng, C. Y. Wang, Temperature jump coefficient for superhydrophobic surfaces, Journal of Heat Transfer 136 (6) (2014) 064501. doi:10.1115/1.4026499.

[41] D. Dressaire, L. Courbin, J. Crest, H. A. Stone, Thin-film fluid flows over microdecorated surfaces: Observation of polygonal hydraulic jumps, Physical Review Letters 102 (2009) 194502. doi:10.1103/ PhysRevLett.102.194503. 